BULLETIN (New Series) OF THE

AMERICAN MATHEMATICAL SOCIETY

Volume 41, Number 2, Pages 151-184

S 0273-0979(04)01001-8

Article electronically published on January 8, 2004

\title{
RECENT ADVANCES IN THE LANGLANDS PROGRAM
}

\author{
EDWARD FRENKEL
}

\begin{abstract}
These are the notes for the lecture given by the author at the "Mathematical Current Events" Special Session of the AMS meeting in Baltimore on January 17, 2003. Topics reviewed include the Langlands correspondence for $G L(n)$ in the function field case and its proof by V. Drinfeld and L. Lafforgue; the geometric Langlands correspondence for $G L(n)$ and its proof by D. Gaitsgory, K. Vilonen and the author; and the work of A. Beilinson and V. Drinfeld on the quantization of the Hitchin system and the Langlands correspondence for an arbitrary semisimple algebraic group.
\end{abstract}

\section{INTRODUCTION}

1.1. The Langlands Program emerged in the late 60's in the form of a series of farreaching conjectures tying together seemingly unrelated concepts in number theory, algebraic geometry, and the theory of automorphic forms [L1]. To motivate it, recall the classical Kronecker-Weber theorem which describes the maximal abelian extension $\mathbb{Q}^{\mathrm{ab}}$ of the field $\mathbb{Q}$ of rational numbers (i.e., the maximal extension of $\mathbb{Q}$ whose Galois group is abelian). This theorem states that $\mathbb{Q}^{\text {ab }}$ is obtained by adjoining to $\mathbb{Q}$ all roots of unity; in other words, $\mathbb{Q}^{\text {ab }}$ is the union of all cyclotomic fields $\mathbb{Q}\left(\zeta_{N}\right)$ obtained by adjoining to $\mathbb{Q}$ a primitive $N$ th root of unity $\zeta_{N}$.

The Galois group $\operatorname{Gal}\left(\mathbb{Q}\left(\zeta_{N}\right) / \mathbb{Q}\right)$ of automorphisms of $\mathbb{Q}\left(\zeta_{N}\right)$ preserving $\mathbb{Q} \subset$ $\mathbb{Q}\left(\zeta_{N}\right)$ is isomorphic to the group $(\mathbb{Z} / N \mathbb{Z})^{\times}$of units of the ring $\mathbb{Z} / N \mathbb{Z}$. Indeed, each element $m \in(\mathbb{Z} / N \mathbb{Z})^{\times}$, viewed as an integer relatively prime to $N$, gives rise to an automorphism of $\mathbb{Q}\left(\zeta_{N}\right)$ which sends $\zeta_{N}$ to $\zeta_{N}^{m}$. Therefore we obtain that the Galois $\operatorname{group} \operatorname{Gal}\left(\mathbb{Q}^{\mathrm{ab}} / \mathbb{Q}\right)$, or, equivalently, the maximal abelian quotient of $\operatorname{Gal}(\overline{\mathbb{Q}} / \mathbb{Q})$, where $\mathbb{Q}$ is an algebraic closure of $\mathbb{Q}$, is isomorphic to the projective limit of the groups $(\mathbb{Z} / N \mathbb{Z})^{\times}$with respect to the system of surjections $(\mathbb{Z} / N \mathbb{Z})^{\times} \rightarrow(\mathbb{Z} / M \mathbb{Z})^{\times}$ for $M$ dividing $N$. This projective limit is nothing but the direct product of the multiplicative groups of the rings of $p$-adic integers, $\mathbb{Z}_{p}^{\times}$, where $p$ runs over the set of all primes. Thus, we obtain that

$$
\operatorname{Gal}\left(\mathbb{Q}^{\mathrm{ab}} / \mathbb{Q}\right) \simeq \prod_{p} \mathbb{Z}_{p}^{\times} .
$$

The abelian class field theory gives a similar description for the maximal abelian quotient $\operatorname{Gal}\left(F^{\mathrm{ab}} / F\right)$ of the Galois group $\operatorname{Gal}(\bar{F} / F)$, where $F$ is an arbitrary global field, i.e., a finite extension of $\mathbb{Q}$ (number field), or the field of rational functions

Received by the editors May 1, 2003, and, in revised form, September 22, 2003

2000 Mathematics Subject Classification. Primary 11R39, 14D20.

Partially supported by grants from the Packard Foundation and the NSF.

Notes for the lecture at the "Mathematical Current Events" Special Session at the AMS meeting in Baltimore, January 17, 2003. 
on a smooth projective curve defined over a finite field (function field). Namely, $\operatorname{Gal}\left(F^{\mathrm{ab}} / F\right)$ is almost isomorphic to the quotient $F^{\times} \backslash \mathbb{A}_{F}^{\times}$, where $\mathbb{A}_{F}$ is the ring of adèles of $F$, a subring in the direct product of all completions of $F$ (see Section 2). Here we use the word "almost" because we need to take the group of components of this quotient if $F$ is a number field, or its profinite completion if $F$ is a function field.

When $F=\mathbb{Q}$ the ring $\mathbb{A}_{\mathbb{Q}}$ is a subring of the direct product of the fields $\mathbb{Q}_{p}$ of $p$ adic numbers and the field $\mathbb{R}$ of real numbers, and the quotient $F^{\times} \backslash \mathbb{A}_{F}^{\times}$is isomorphic to $\mathbb{R}_{>0} \times \prod_{p} \mathbb{Z}_{p}^{\times}$. Hence the group of its components is $\prod_{p} \mathbb{Z}_{p}^{\times}$, in agreement with the Kronecker-Weber theorem.

One can obtain complete information about the maximal abelian quotient of a group by considering its one-dimensional representations. The above statement of the abelian class field theory may then be reformulated as saying that onedimensional representations of $\operatorname{Gal}(\bar{F} / F)$ are essentially in bijection with onedimensional representations of the abelian group $\mathbb{A}_{F}^{\times}=G L_{1}\left(\mathbb{A}_{F}\right)$ which occur in the space of functions on $F^{\times} \backslash \mathbb{A}_{F}^{\times}=G L_{1}(F) \backslash G L_{1}\left(\mathbb{A}_{F}\right)$. A marvelous insight of Robert Langlands was to conjecture that there exists a similar description of $n$-dimensional representations of $\operatorname{Gal}(\bar{F} / F)$. Namely, he proposed that those may be related to irreducible representations of the group $G L_{n}\left(\mathbb{A}_{F}\right)$ which are automorphic, that is those occurring in the space of functions on the quotient $G L_{n}(F) \backslash G L_{n}\left(\mathbb{A}_{F}\right)$. This relation is now called the Langlands correspondence 1

1.2. At this point one might ask, why is it important to know what the $n^{-}$ dimensional representations of the Galois group look like, and why is it useful to relate them to things like automorphic representations? There are indeed many reasons for that. First of all, it should be remarked that according to the Tannakian philosophy, one can reconstruct a group from the category of its finite-dimensional representations, equipped with the structure of the tensor product. Therefore looking at $n$-dimensional representations of the Galois group is a natural step towards understanding its structure. But even more importantly, one finds many interesting representations of Galois groups in "nature". For example, the group $\operatorname{Gal}(\overline{\mathbb{Q}} / \mathbb{Q})$ will act on the geometric invariants (such as the étale cohomologies) of an algebraic variety defined over $\mathbb{Q}$. Thus, if we take an elliptic curve $E$ over $\mathbb{Q}$, then we will obtain a two-dimensional Galois representation on its first étale cohomology. This representation contains a lot of important information about the curve $E$, such as the number of points of $E$ over $\mathbb{Z} / p \mathbb{Z}$ for various primes $p$.

The point is that the Langlands correspondence is supposed to relate $n$-dimensional Galois representations to automorphic representations of $G L_{n}\left(\mathbb{A}_{F}\right)$ in such a way that the data on the Galois side, such as the number of points of $E(\mathbb{Z} / p \mathbb{Z})$, are translated into something more tractable on the automorphic side, such as the coefficients in the $q$-expansion of the modular forms that encapsulate automorphic representations of $G L_{2}\left(\mathbb{A}_{\mathbb{Q}}\right)$.

\footnotetext{
${ }^{1}$ The above correspondence is a natural starting point for this review, but we would like to emphasize the fact that the general Langlands correspondence (also known as the functoriality principle; see e.g. [A2]) is a much more general and intricate statement. This general correspondence will, however, remain beyond the scope of this article.
} 
More precisely, one asks that under the Langlands correspondence certain natural invariants attached to the Galois representations and to the automorphic representations be matched. These invariants are the Frobenius conjugacy classes on the Galois side and the Hecke eigenvalues on the automorphic side. Let us review them briefly. To an $n$-dimensional representation $\sigma$ of $\operatorname{Gal}(\bar{F} / F)$ that is almost everywhere unramified one attaches a collection of conjugacy classes in the group $G L_{n}$ for all but finitely many points $x$ of the curve $X$ if $F=\mathbb{F}_{q}(X)$ (or primes of the ring of integers of $F$ if $F$ is a number field). These are the images of the Frobenius elements in $\operatorname{Gal}(\bar{F} / F)$ (see Section 2.1). The eigenvalues of these conjugacy classes are then represented by unordered $n$-tuples of numbers $\left(z_{1}\left(\sigma_{x}\right), \ldots, z_{n}\left(\sigma_{x}\right)\right)$. On the other hand, to an automorphic representation $\pi$ of $G L_{n}(\mathbb{A})$ one attaches a collection of eigenvalues of the so-called Hecke operators (see Section 2.2), which may also be encoded by unordered collections of $n$-tuples of numbers $\left(z_{1}\left(\pi_{x}\right), \ldots, z_{n}\left(\pi_{x}\right)\right)$ for all but finitely many $x \in X$. If under the Langlands correspondence we have

$$
\pi \longleftrightarrow \sigma
$$

then we should have

$$
\left(z_{1}\left(\pi_{x}\right), \ldots, z_{n}\left(\pi_{x}\right)\right)=\left(z_{1}\left(\sigma_{x}\right), \ldots, z_{n}\left(\sigma_{x}\right)\right)
$$

for all but finitely many $x \in X$ (up to permutation).

The Frobenius eigenvalues and the Hecke eigenvalues may be converted into analytic objects called the $L$-functions. Then the matching of these eigenvalues becomes the statement that the $L$-functions corresponding to $\pi$ and $\sigma$ are equal.

It is instructive to consider what this matching means in the simplest example when $F=\mathbb{Q}$ and $n=1$. In this case the Langlands correspondence comes from the isomorphism between the abelian quotient of the Galois group $\operatorname{Gal}\left(\mathbb{Q}^{\text {ab }} / \mathbb{Q}\right)$ and the group of components of $\mathbb{Q}^{\times} \backslash \mathbb{A}_{\mathbb{Q}}^{\times}$. The matching condition (1.1) then means that the Frobenius element $\operatorname{Fr}_{p}$ corresponding to the prime $p \in \mathbb{Z}$ in $\operatorname{Gal}\left(\mathbb{Q}^{\mathrm{ab}} / \mathbb{Q}\right)$ (more precisely, of its quotient unramified at $p$ ) goes under this isomorphism to the image of the adèle $(1, \ldots, 1, p, 1, \ldots)$, with $p$ being in the factor $\mathbb{Q}_{p}^{\times}$, in the group of components of $\mathbb{Q}^{\times} \backslash \mathbb{A}_{\mathbb{Q}}^{\times}$.

Let us represent the latter group as $\lim (\mathbb{Z} / N \mathbb{Z})^{\times}$, where $(\mathbb{Z} / N \mathbb{Z})^{\times}$is considered as the Galois group $\operatorname{Gal}\left(\mathbb{Q}\left(\zeta_{N}\right) / \mathbb{Q}\right)$ of the cyclotomic field $\mathbb{Q}\left(\zeta_{N}\right)$. Then the above statement translates into the statement as to what is the image of the Frobenius conjugacy class $\operatorname{Fr}_{p}$ in $\operatorname{Gal}\left(\mathbb{Q}\left(\zeta_{N}\right) / \mathbb{Q}\right)=(\mathbb{Z} / N \mathbb{Z})^{\times}$. It says that this image is equal to $p \bmod N$; note that this makes sense only if $p$ does not divide $N$, which is precisely the condition that $p$ is unramified in $\mathbb{Q}\left(\zeta_{N}\right)$. Since the Frobenius element $\operatorname{Fr}_{p}$ comes from the automorphism $y \mapsto y^{p}$ of the finite field $\mathbb{F}_{p}$, it is not surprising that it corresponds to the automorphism of $\mathbb{Q}\left(\zeta_{N}\right)$ sending $\zeta_{N}$ to $\zeta_{N}^{p}$.

Thus, we find that the order of $\operatorname{Fr}_{p}$ in $\operatorname{Gal}\left(\mathbb{Q}\left(\zeta_{N}\right) / \mathbb{Q}\right)$ is equal to the order of $p$ in $(\mathbb{Z} / N \mathbb{Z})^{\times}$. Therefore we may figure out how $p$ splits in the ring $\mathbb{Z}\left[\zeta_{N}\right]$. If the order of $\operatorname{Fr}_{p}$ is equal to $d$ and $(p)=\mathcal{P}_{1} \ldots \mathcal{P}_{r}$, where the $\mathcal{P}_{i}$ 's are prime ideals in $\mathbb{Z}\left[\zeta_{N}\right]$, then the residue field of each of the $\mathcal{P}_{i}$ 's should be an extension of $\mathbb{F}_{p}$ of degree $d$, and therefore $r=\varphi(N) / d$, where $\varphi(N)=\left|(\mathbb{Z} / N \mathbb{Z})^{\times}\right|$. So we obtain an application of the matching (1.1) in the case $n=1$ to the problem of the splitting of primes. For instance, if $N=4$ we obtain that $p$ splits in $\mathbb{Z}[i]$; i.e., $p$ may be represented as a sum of two squares of integers,

$$
p=(a+b i)(a-b i)=a^{2}+b^{2},
$$


if and only if $p \equiv 1 \bmod 4$, which is the statement of one of Fermat's theorems (see Ge] for more details).

Likewise, in the case of two-dimensional Galois representations $\sigma$ arising from the first étale cohomology of an elliptic curve $E$ over $\mathbb{Z}$, the eigenvalues of $\operatorname{Fr}_{p}$ (which are well-defined for all $p$ 's that do not divide the conductor of $E$ ) contain information about the number of points of $E$ over $\mathbb{Z} / p \mathbb{Z}$. Suppose now that $\pi$ is an automorphic representation of $G L_{2}(\mathbb{A})$ attached to $\sigma$ via the Langlands correspondence. One assigns to $\pi$ in a natural way a modular form on the upper-half plane (see, e.g., $\mathrm{Ge}, \mathrm{Mu})$. Then the matching condition (1.1) relates the numbers of points of $E(\mathbb{Z} / p \mathbb{Z})$ to the coefficients in the $q$-expansion of this modular form. The existence of $\pi$ (and hence of the corresponding modular form) now becomes the statement of the Taniyama-Shimura (or Taniyama-Shimura-Weil) conjecture that has recently been proved by A. Wiles and others (it implies Fermat's last theorem). One obtains similar statements by analyzing from the point of view of the Langlands philosophy the Galois representations coming from other algebraic varieties, or more general motives.

1.3. While in the number field case the Langlands correspondence has been established only in special cases such as the one expressed by the Taniyama-Shimura conjecture, in the function field case the Langlands conjecture is now a theorem. It was proved in the 80 's by V. Drinfeld in the case when $n=2$ and recently by L. Lafforgue for an arbitrary $n$ in a monumental effort for which both of them have been awarded Fields Medals.

In these notes we will focus on the Langlands correspondence in the function field case. We will start by giving a precise formulation of the Langlands conjecture in this case (see Section 2). Then in Section 3 we will describe some of the ideas involved in the proof of this conjecture given by Drinfeld and Lafforgue.

After that we will discuss in Section 4 the geometric Langlands correspondence. This is a geometric version of the Langlands conjecture (available only in the function field case). It comes from the observation that $n$-dimensional representations of $\operatorname{Gal}(\bar{F} / F)$, where $F=\mathbb{F}_{q}(X)$, may be viewed geometrically as rank $n$ local systems on $X$. Such objects make sense both when $X$ is a curve over a finite field and over $\mathbb{C}$. Thus, we may transport the objects on the Galois side of the Langlands correspondence to the realm of complex algebraic geometry.

It turns out that one may replace the automorphic representations of $G L_{n}(\mathbb{A})$ by geometric objects as well. Those are the Hecke eigensheaves, which are certain sheaves on the moduli space of rank $n$ bundles on the curve. The geometric Langlands conjecture states, roughly, that to any rank $n$ local system $E$ on $X$ one may associate a Hecke eigensheaf whose "eigenvalues" are expressed in terms of $E$. The advantage of this formulation is that it makes sense for curves defined not only over finite fields but also over the field of complex numbers. Hence one can use the machinery of complex algebraic geometry to gain new insights into the nature of the Langlands correspondence. For example, for $n=1$ the geometric Langlands conjecture states that to each rank one local system $E$ on $X$ one can associate a Hecke eigensheaf on the Jacobean variety of $X$. It turns out that when $X$ is a curve over $\mathbb{C}$, this correspondence is best understood in the framework of the Fourier-Mukai transform (see Section 6.2). 
The geometric Langlands conjecture for $G L_{n}$ has recently been proved in [FGV], Ga] following earlier works of P. Deligne, V. Drinfeld [Dr4] and G. Laumon [La2], La3. In Section 4 we will give an overview of this proof.

Then in Section 5 we will discuss how to formulate the Langlands correspondence for an arbitrary reductive group $G$ instead of $G L_{n}$, both in the classical and the geometric settings. This is where one of the most beautiful and mysterious concepts, that of the Langlands dual group, enters the subject. Finally, we will describe in Section 6 the work BD of A. Beilinson and V. Drinfeld in which part of this conjecture has been proved for an arbitrary semi-simple Lie group $G$ over $\mathbb{C}$. Their proof uses the geometry of moduli spaces of bundles on curves as well as representation theory of affine Kac-Moody algebras.

In the last thirty years the subject of the Langlands correspondence has evolved into a vast and multifaceted field. In a short review it is impossible to even glance over its main ideas and trends. In particular, we will not mention such remarkable recent achievements as the proof of the local Langlands conjectures for $G L_{n}$, both in the function field and number field settings, given in [LSR] and [HT], [He], respectively (in that regard we refer the reader to Carayol's talk at Séminaire Bourbaki C]).

A number of wonderful reviews of the Langlands Program are available at present, and I would like to mention some of them. The papers $\mathrm{A1}$, $\mathrm{A2}$, $\mathrm{Ge}, \mathrm{Kn}, \mathrm{Mu}$ contain general overviews of the Langlands Program which are informative and fun to read. The reports $[\mathrm{Kaz}]$ and $[\mathrm{HK}]$ give a clear and concise review of the work of Drinfeld in the case of $G L_{2}$. For excellent expositions of Lafforgue's work and the geometric Langlands correspondence we refer the reader to Laumon's talks at Séminaire Bourbaki, [La5] and [La6], respectively. Finally, the recent article [L2] offers a unique perspective on the field by its creator "thirty years later".

\section{The Statement of the Langlands COnjeCture in the FUNCTION FIELD CASE}

Let $X$ be a smooth projective curve over a finite field $k=\mathbb{F}_{q}$ with $q$ elements. We will denote by $\bar{X}$ the corresponding curve over the algebraic closure $\overline{\mathbb{F}}_{q}$ of $\mathbb{F}_{q}$, $\bar{X}=X \underset{\text { Spec }_{q}}{\times} \operatorname{Spec} \overline{\mathbb{F}}_{q}$. We will assume that $\bar{X}$ is connected. Let $F=\mathbb{F}_{q}(X)$ be the field of rational functions on $X$, and fix its separable closure $\bar{F}$. We denote by $G_{F}$ the Galois group of $F$, i.e., the group of automorphisms of $\bar{F}$ preserving $F$.

For any closed point $x$ of $X$, we denote by $F_{x}$ the completion of $F$ at $x$ and by $\mathcal{O}_{x}$ its ring of integers. If we pick a rational function $t_{x}$ on $X$ which vanishes at $x$ to order one, then we obtain isomorphisms $F_{x} \simeq k_{x}\left(\left(t_{x}\right)\right)$ and $\mathcal{O}_{x} \simeq k_{x}\left[\left[t_{x}\right]\right]$, where $k_{x}$ is the residue field of $x$ (the quotient of the local ring $\mathcal{O}_{x}$ by its maximal ideal); this field is a finite extension of the base field $k$ and hence is isomorphic to $\mathbb{F}_{q_{x}}$, where $q_{x}=q^{\operatorname{deg} x}$. The ring $\mathbb{A}=\mathbb{A}_{F}$ of adèles of $F$ is by definition the restricted product of the fields $F_{x}$, where $x$ runs over the set $|X|$ of all closed points of $X$. The word "restricted" means that we consider only the collections $\left(f_{x}\right)_{x \in|X|}$ of elements of $F_{x}$ in which $f_{x} \in \mathcal{O}_{x}$ for all but finitely many $x$. The ring $\mathbb{A}$ contains the field $F$, which is embedded into $\mathbb{A}$ diagonally, by taking the expansions of rational functions on $X$ at all points.

Roughly speaking, the Langlands conjecture states that there is a bijection between the set of equivalence classes of $n$-dimensional representations of $G_{F}$ and the 
set of equivalence classes of irreducible automorphic representations of $G L_{n}(\mathbb{A})$, i.e., those which may be realized in a certain space of functions on the quotient $G L_{n}(F) \backslash G L_{n}(\mathbb{A})$. In order to make a precise formulation of the conjecture, we need to explain what all of this means. We will also restrict ourselves to irreducible $n$-dimensional representations of $G_{F}$. Those will correspond to the so-called cuspidal representations of $G L_{n}(\mathbb{A})$. In addition, using the abelian class field theory, we can without loss of generality consider only those representations of $G_{F}$ whose determinant has finite order, and those representations of $G L_{n}(\mathbb{A})$ whose central character has finite order (a general representation may be obtained by tensoring a representation of this type with a one-dimensional representation). Let us now give the precise definitions.

2.1. Galois representations. Consider the Galois group $G_{F}$. It is instructive to think of it as a kind of fundamental group of $X$. Indeed, if $Y \rightarrow X$ is a covering of $X$, then the field $k(Y)$ of rational functions on $Y$ is an extension of the field $F=k(X)$ of rational functions on $X$, and the Galois group $\operatorname{Gal}(k(Y) / k(X))$ may be viewed as the group of "deck transformations" of the cover. If our cover is unramified, then this group may be identified with a quotient of the fundamental group of $X$. Otherwise, this group is isomorphic to a quotient of the fundamental group of $X$ without the ramification points. The Galois group $G_{F}$ itself may be viewed as the group of "deck transformations" of the maximal (ramified) cover of $X$.

Let us pick a point $\bar{x}$ of this cover lying over a fixed point $x \in|X|$. The subgroup of $G_{F}$ preserving $\bar{x}$ is called the decomposition group. If we make a different choice of $\bar{x}$, it gets conjugated in $G_{F}$. Therefore we obtain a subgroup of $G_{F}$ defined up to conjugation. We denote it by $D_{x}$. This group is in fact isomorphic to the Galois group $\operatorname{Gal}\left(\bar{F}_{x} / F_{x}\right)$, and we have a natural homomorphism $D_{x} \rightarrow$ $\operatorname{Gal}\left(\bar{k}_{x} / k_{x}\right)$, whose kernel is called the inertia subgroup and is denoted by $I_{x}$. The Galois group $\operatorname{Gal}\left(\bar{k}_{x} / k_{x}\right)$ has a very simple description: it contains the (geometric) Frobenius element $\mathrm{Fr}_{x}$, which is defined to be the inverse of the automorphism $y \mapsto y^{q_{x}}$ of $\bar{k}_{x}=\overline{\mathbb{F}}_{q_{x}}$, and $\operatorname{Gal}\left(\bar{k}_{x} / k_{x}\right)$ is equal to the profinite completion of the group $\mathbb{Z}$ generated by this element.

A homomorphism $\sigma$ from $G_{F}$ to another group $H$ is called unramified at $x$ if $I_{x}$ lies in the kernel of $\sigma$ (this condition is independent of the choice of $\bar{x}$ ). In this case $\mathrm{Fr}_{x}$ gives rise to a well-defined conjugacy class in $H$, denoted by $\sigma\left(\mathrm{Fr}_{x}\right)$.

The group $G_{F}$ is a profinite group, equipped with the Krull topology in which the base of open neighborhoods of the identity is formed by normal subgroups of finite index. Therefore a continuous finite-dimensional complex representation of $G_{F}$ necessarily factors through a finite quotient of $G_{F}$. To obtain a larger class of Galois representations we replace $\mathbb{C}$ with the field $\mathbb{Q}_{\ell}$ of $\ell$-adic numbers, where $\ell$ is a prime that does not divide $q$. Denote by $\overline{\mathbb{Q}}_{\ell}$ the algebraic closure of $\mathbb{Q}_{\ell}$. By an $n$-dimensional $\ell$-adic representation of $G_{F}$ we will understand a homomorphism $\sigma: G_{F} \rightarrow G L_{n}\left(\overline{\mathbb{Q}}_{\ell}\right)$ which satisfies the following conditions:

(1) there exists a finite extension $E \subset \overline{\mathbb{Q}}_{\ell}$ of $\mathbb{Q}_{\ell}$ such that $\sigma$ factors through a homomorphism $G_{F} \rightarrow G L_{n}(E)$, which is continuous with respect to the Krull topology on $G_{F}$ and the $\ell$-adic topology on $G L_{n}(E)$;

(2) it is unramified at all but finitely many $x \in|X|$. 
Let $\mathcal{G}_{n}$ be the set of equivalence classes of irreducible $n$-dimensional $\ell$-adic representations of $G_{F}$ such that the image of $\operatorname{det}(\sigma)$ is a finite group. Given such a representation, we consider the collection of the Frobenius conjugacy classes $\left\{\sigma\left(\operatorname{Fr}_{x}\right)\right\}$ in $G L_{n}\left(\overline{\mathbb{Q}}_{\ell}\right)$ and the collection of their eigenvalues (defined up to permutation), which we denote by $\left\{\left(z_{1}\left(\sigma_{x}\right), \ldots, z_{n}\left(\sigma_{x}\right)\right)\right\}$, for all $x \in|X|$ where $\sigma$ is unramified. Chebotarev's density theorem implies the following remarkable result: if two $\ell$-adic representations are such that their collections of the Frobenius conjugacy classes coincide for all but finitely many points $x \in|X|$, then these representations are equivalent.

2.2. Automorphic representations. Consider now the group $G L_{n}(\mathbb{A})$. It carries a Haar measure normalized in such a way that the volume of the subgroup $G L_{n}\left(\mathcal{O}_{x}\right)$ is equal to 1 for all $x \in|X|$. Note that $G L_{n}(F)$ is naturally a subgroup of $G L_{n}(\mathbb{A})$. A function $\varphi: G L_{n}(\mathbb{A}) \rightarrow \overline{\mathbb{Q}}_{\ell}$ is called cuspidal automorphic if it satisfies the following conditions:

(1) it is left $G L_{n}(F)$-invariant;

(2) it is right invariant with respect to an open subgroup of $G L_{n}(\mathbb{A})$;

(3) there exists an element $a \in \mathbb{A}^{\times}$of non-zero degree such that $\varphi(a g)=\varphi(g)$ for all $g \in G L_{n}(\mathbb{A})$;

(4) if $N_{n_{1}, n_{2}}$ is the unipotent radical of the standard parabolic subgroup $P_{n_{1}, n_{2}}$ of $G L_{n}$ corresponding to the partition $n=n_{1}+n_{2}$ with $n_{1}, n_{2}>0$, then

$$
\int_{N_{n_{1}, n_{2}}(F) \backslash N_{n_{1}, n_{2}}(\mathbb{A})} \varphi(u g) d u=0, \quad \forall g \in G L_{n}(\mathbb{A}) .
$$

Denote the vector space of cuspidal automorphic functions on $G L_{n}(\mathbb{A})$ by $\mathcal{C}_{\text {cusp }}$. The group $G L_{n}(\mathbb{A})$ acts on $\mathcal{C}_{\text {cusp }}$, and under this action $\mathcal{C}_{\text {cusp }}$ decomposes into a direct sum of irreducible representations. These representations are called irreducible cuspidal automorphic representations of $G L_{n}(\mathbb{A})$. A theorem due to PiatetskiShapiro [PS1] and Shalika [Sh] says that each of them enters $\mathcal{C}_{\text {cusp }}$ with multiplicity one. We denote the set of equivalence classes of these representations by $\mathcal{A}_{n}$.

Remark 2.1. If $\pi_{1}$ and $\pi_{2}$ are irreducible representations of $G L_{n_{1}}(\mathbb{A})$ and $G L_{n_{2}}(\mathbb{A})$, respectively, where $n_{1}+n_{2}=n$, then we may extend trivially the representation $\pi_{1} \otimes \pi_{2}$ of $G L_{n_{1}} \times G L_{n_{2}}$ to the parabolic subgroup $P_{n_{1}, n_{2}}(\mathbb{A})$ and consider the induced representation of $G L_{n}(\mathbb{A})$. It is easy to see that irreducible quotients of this representation cannot be realized in the space $\mathcal{C}_{\text {cusp }}$ because of the cuspidality condition (4). In fact, this condition is imposed precisely so as to avoid irreducible quotients of induced representations.

We also remark that condition (3) is equivalent to the central character of $\pi$ being of finite order.

Let $\pi$ be an irreducible cuspidal automorphic representation of $G L_{n}(\mathbb{A})$. One can show that it decomposes into a tensor product

$$
\pi=\bigotimes_{x \in|X|}^{\prime} \pi_{x}
$$

where each $\pi_{x}$ is an irreducible representation of $G L_{n}\left(F_{x}\right)$. Furthermore, there is a finite subset $S$ of $|X|$ such that each $\pi_{x}$ with $x \in|X|-S$ is unramified, i.e., contains a non-zero vector $v_{x}$ stable under the maximal compact subgroup $G L_{n}\left(\mathcal{O}_{x}\right)$ of $G L_{n}\left(F_{x}\right)$. This vector is unique up to a scalar, and we will fix it once and for all. 
The space $\bigotimes_{x \in|X|}^{\prime} \pi_{x}$ is by definition the span of all vectors of the form $\bigotimes_{x \in|X|} w_{x}$, where $w_{x} \in \pi_{x}$ and $w_{x}=v_{x}$ for all but finitely many $x \in|X|-S$. Therefore the action of $G L_{n}(\mathbb{A})$ on $\pi$ is well-defined.

Let $\mathcal{H}_{x}$ be the space of compactly supported functions on $G L_{n}\left(F_{x}\right)$ which are bi-invariant with respect to the subgroup $G L_{n}\left(\mathcal{O}_{x}\right)$. This is an algebra with respect to the convolution product, which is called the spherical Hecke algebra. By the Satake correspondence (see Theorem 5.1 below), we have an isomorphism

$$
\mathcal{H}_{x} \simeq \overline{\mathbb{Q}}_{\ell}\left[z_{1}^{ \pm 1}, \ldots, z_{n}^{ \pm 1}\right]^{S_{n}} .
$$

The Hecke algebra $\mathcal{H}_{x}$ naturally acts on any irreducible unramified representation $\pi_{x}$ of $G L_{n}\left(F_{x}\right)$ and preserves the one-dimensional subspace of $G L_{n}\left(\mathcal{O}_{x}\right)$-invariant vectors spanned by $v_{x}$. Hence $\mathcal{H}_{x}$ acts on it via a character, which is nothing but a collection of non-zero numbers $\left(z_{1}\left(\pi_{x}\right), \ldots, z_{n}\left(\pi_{x}\right)\right)$ defined up to permutation. We will call them the Hecke eigenvalues of $\pi$ at $x$. Thus, to each irreducible cuspidal automorphic representation $\pi$ one associates a collection of Hecke eigenvalues $\left\{\left(z_{1}\left(\pi_{x}\right), \ldots, z_{n}\left(\pi_{x}\right)\right)\right\}_{x \in|X|-S}$, defined up to permutation. The strong multiplicity one theorem due to Piatetski-Shapiro PS1] says that this collection determines $\pi$ up to an isomorphism.

2.3. The Langlands correspondence and related results. Now we can state the Langlands conjecture for $G L_{n}$ in the function field case. It has been proved by Drinfeld [Dr1], Dr2] for $n=2$ and by Lafforgue Laf2] for $n>2$.

Theorem 2.2. There is a bijection between the sets $\mathcal{A}_{n}$ and $\mathcal{G}_{n}$ defined above which satisfies the following condition. If $\pi \in \mathcal{A}_{n}$ corresponds to $\sigma \in \mathcal{G}_{n}$, then the sets of points where they are unramified are the same, and for each $x$ from this set we have

$$
\left(z_{1}\left(\pi_{x}\right), \ldots, z_{n}\left(\pi_{x}\right)\right)=\left(z_{1}\left(\sigma_{x}\right), \ldots, z_{n}\left(\sigma_{x}\right)\right)
$$

up to permutation.

In other words, if $\pi$ and $\sigma$ correspond to each other, then the Hecke eigenvalues of $\pi$ coincide with the Frobenius eigenvalues of $\sigma$ at all points where they are unramified.

In addition to the Langlands correspondence, Drinfeld Dr3] (for $n=2$ ) and Lafforgue [Laf1], Laf2] (for $n>2$ ) have also proved the following result.

Theorem 2.3. At all points $x \in|X|$ where $\pi \in \mathcal{A}_{n}$ is unramified, the Hecke eigenvalues $z_{i}\left(\pi_{x}\right)$ of $\pi$ are algebraic numbers with (complex) absolute value equal to 1.

Remark 2.4. The statement saying that $\left|z_{i}\left(\pi_{x}\right)\right|=1$ is called the RamanujanPetersson conjecture. Its classical analogue is the Ramanujan conjecture, proved by Deligne, that the coefficients $\tau(n)$ of the Ramanujan $\tau$-function

$$
q \prod_{m=1}\left(1-q^{m}\right)^{24}=\sum_{n=1}^{\infty} \tau(n) q^{n}
$$

satisfy the relation $|\tau(p)| \leq 2 p^{11 / 2}$ for a prime $p$. It turns out that the numbers $\tau(p)$ may be written in the form

$$
\tau(p)=p^{11 / 2}\left(\alpha_{p}+\beta_{p}\right)
$$


where $\alpha_{p}$ and $\beta_{p}=\bar{\alpha}_{p}$ are the Hecke eigenvalues of an automorphic representation of $G L_{2}\left(\mathbb{A}_{\mathbb{Q}}\right)$ corresponding to the modular form $\tau(q)$. Therefore the Ramanujan conjecture is equivalent to the statement that $\left|\alpha_{p}\right|=\left|\beta_{p}\right|=1$, which is analogous to the condition appearing in Theorem 2.3 .

Theorem 2.3 has a stunning corollary, known as the Deligne purity conjecture De2]. Let $V$ be a normal algebraic variety over $\mathbb{F}_{q}$. Then one defines, in the same way as above in the case of curves, $\ell$-adic representations of the Galois group $\operatorname{Gal}(\bar{F} / F)$, where $F$ is the field of rational functions on $V$ and $\bar{F}$ is its separable closure. The following theorem is proved by reducing the statement to the case where $V$ is a curve and applying Theorems 2.2 and 2.3 .

Theorem 2.5. Let $\sigma$ be any irreducible $\ell$-adic representation of $\operatorname{Gal}(\bar{F} / F)$, which is everywhere unramified and has determinant of finite order. Then the eigenvalues of the Frobenius conjugacy classes at all closed points of $V$ are algebraic numbers with (complex) absolute value 1.

\section{Elements of the PROOF of the LANGlands ConjeCture}

In this section we discuss some of the ideas and constructions involved in the proof of the Langlands correspondence, Theorem 2.2 by Drinfeld and Lafforgue. In the first approximation, one can say that the main idea is to realize this correspondence in the cohomology of a certain moduli space of vector bundles on $X$ with some additional structures, called "shtukas", and to use the Grothendieck-Lefschetz formula to relate the traces of Hecke correspondences acting on this cohomology with the numbers of fixed points of these correspondences acting on the moduli space.

3.1. From automorphic functions to vector bundles. Recall that automorphic representations of $G L_{n}(\mathbb{A})$ are realized in the space $\mathcal{C}_{\text {cusp }}$ of functions on the quotient $G L_{n}(F) \backslash G L_{n}(\mathbb{A})$. Suppose that we are given an automorphic representation $\pi$ of $G L_{n}(\mathbb{A})$ which is unramified at all points of $X$. Then the space of $G L_{n}(\mathcal{O})$-invariants in $\pi$, where $\mathcal{O}=\prod_{x \in|X|} \mathcal{O}_{x}$, is one-dimensional, spanned by the vector

$$
v=\bigotimes_{x \in|X|} v_{x}
$$

where $v_{x}$ is defined in Section 2.2 Hence $v$ gives rise to a $G L_{n}(\mathcal{O})$-invariant function on $G L_{n}(F) \backslash G L_{n}(\mathbb{A})$, or equivalently, a function on the double quotient

$$
G L_{n}(F) \backslash G L_{n}(\mathbb{A}) / G L_{n}(\mathcal{O}) .
$$

The following key observation is due to A. Weil.

Lemma 3.1. There is a bijection between the set $G L_{n}(F) \backslash G L_{n}(\mathbb{A}) / G L_{n}(\mathcal{O})$ and the set of isomorphism classes of rank $n$ vector bundles on $X$.

Proof. Any rank $n$ bundle on $X$ may be trivialized on the formal disc $D_{x}=\operatorname{Spec} \mathcal{O}_{x}$ around each point $x \in|X|$ and over $X-S$, where $S$ is a sufficiently large subset of $|X|$, hence over the generic point $X_{\text {gen }}=\operatorname{Spec} F$ of $X$.

Let $B$ be the set of isomorphism classes of the data $\left(\mathcal{M}, \varphi_{\text {gen }},\left(\varphi_{x}\right)\right)$, where $\mathcal{M}$ is a rank $n$ bundle on $X$, and $\varphi_{\text {gen }}$ and $\varphi_{x}, x \in|X|$, are the trivializations of $\mathcal{M}$ over the generic point of $X$ and the formal discs $D_{x}$, respectively, i.e., isomorphisms $\varphi_{\text {gen }}: F^{n} \stackrel{\sim}{\rightarrow} \Gamma\left(X_{\text {gen }}, \mathcal{M}\right), \varphi_{x}: \mathcal{O}_{x}^{n} \stackrel{\sim}{\rightarrow} \Gamma\left(D_{x}, \mathcal{M}\right)$. The restrictions of $\varphi_{\text {gen }}$ and $\varphi_{x}$ to the punctured disc $D_{x}^{\times}=\operatorname{Spec} \mathcal{K}_{x}$ then give us two different trivializations of $\left.\mathcal{M}\right|_{D_{x}^{\times}}$, 
which we denote by the same symbols. Let $g_{x}=\varphi_{\text {gen }}^{-1} \circ \varphi_{x}$ be the corresponding transition function, which is an element of $G L_{n}\left(\mathcal{K}_{x}\right)$.

Consider the map $b: B \rightarrow G L_{n}(\mathbb{A})$ sending $\left(\mathcal{M}, \varphi_{\text {gen }},\left(\varphi_{x}\right)\right)$ to $\left(g_{x}\right)_{x \in|X|} \in$ $G L_{n}(\mathbb{A})$. It is easy to see that this map is a bijection. Therefore there is bijection between the set of isomorphism classes of rank $n$ bundles on $X$ and the quotient of $G L_{n}(\mathbb{A})$ by the equivalence relations corresponding to changes of the trivializations $\varphi_{\text {gen }}$ and $\varphi_{x}, x \in|X|$. These equivalence relations amount to the left action of $G L_{n}(F)$ and the right action of $G L_{n}(\mathcal{O})$ on $G L_{n}(\mathbb{A})$, respectively. Hence we obtain the statement of the lemma.

In order to apply the machinery of algebraic geometry one needs to interpret sets like $G L_{n}(F) \backslash G L_{n}(\mathbb{A}) / G L_{n}(\mathcal{O})$ as sets of points of algebraic varieties over $\mathbb{F}_{q}$. Once this is done, one can use things like cohomology groups of varieties and techniques like the Lefschetz fixed point formula. The above result gives precisely such an interpretation of the double quotient $G L_{n}(F) \backslash G L_{n}(\mathbb{A}) / G L_{n}(\mathcal{O})$. Namely, we obtain that it is the set of $\mathbb{F}_{q}$-points of the moduli space $\operatorname{Bun}_{n}$ of rank $n$ vector bundles on $X$. To be precise, Bun $_{n}$ is not an algebraic variety, but an algebraic stack, which means, roughly speaking, that it looks locally like an algebraic variety quotiented out by the action of an algebraic group (these actions are not free, and therefore the quotient is no longer an algebraic variety); see [LMB] for the precise definition. But for our purposes this turns out to be sufficient.

3.2. Hecke correspondences. Another important observation is the interpretation of the spherical Hecke algebra in terms of Hecke correspondences in Bun $_{n} \times$ $\operatorname{Bun}_{n}$. The spherical Hecke algebra $\mathcal{H}=\bigotimes_{x \in|X|} \mathcal{H}_{x}$ naturally acts on the space of functions on $G L_{n}(F) \backslash G L_{n}(\mathbb{A}) / G L_{n}(\mathcal{O})$, where it preserves the one-dimensional subspaces $\pi^{G L_{n}(\mathcal{O})}$ of $G L_{n}(\mathcal{O})$-invariants in the unramified irreducible representations $\pi$ of $G L_{n}(\mathbb{A})$. Let us describe the algebra $\mathcal{H}_{x}$ in more detail. According to formula (2.1), $\mathcal{H}_{x}$ is isomorphic to the algebra generated by the elementary symmetric functions in $z_{1}, \ldots, z_{n}$, which we denote by $H_{1, x}, \ldots, H_{n, x}$, and $H_{n, x}^{-1}$. The action of the operator $H_{i, x}$ on the space of functions on $G L_{n}(F) \backslash G L_{n}(\mathbb{A}) / G L_{n}(\mathcal{O})$ is given by the following integral operators:

$$
\left(H_{i, x} \cdot f\right)(g)=\int_{M_{n}^{i}\left(\mathcal{O}_{x}\right)} f(g h) d h,
$$

where

$$
M_{n}^{i}\left(\mathcal{O}_{x}\right)=G L_{n}\left(\mathcal{O}_{x}\right) \cdot \mathcal{D}_{x}^{i} \cdot G L_{n}\left(\mathcal{O}_{x}\right) \subset G L_{n}\left(F_{x}\right) \subset G L_{n}(\mathbb{A}),
$$

$\mathcal{D}_{x}^{i}$ is the diagonal matrix whose first $i$ entries are equal to $t_{x}$ (a uniformizer at $x$ ), and whose remaining $n-i$ entries are equal to 1 .

Now define the $i$ th Hecke correspondence $\mathcal{H e c k e}_{i}$ (in what follows we will use the same notation for a vector bundle and for the sheaf of its sections, which is a locally free coherent sheaf). It is the moduli space of quadruples

$$
\left(\mathcal{M}, \mathcal{M}^{\prime}, x, \beta: \mathcal{M}^{\prime} \hookrightarrow \mathcal{M}\right)
$$

where $\mathcal{M}^{\prime}, \mathcal{M} \in \operatorname{Bun}_{n}, x \in|X|$, and $\beta$ is the embedding of the corresponding coherent sheaves of sections $\beta: \mathcal{M}^{\prime} \hookrightarrow \mathcal{M}$ such that $\mathcal{M} / \mathcal{M}^{\prime}$ is supported at $x$ and is isomorphic to the direct sum of $i$ copies of the skyscraper sheaf $\mathcal{O}_{x}=\mathcal{O}_{X} / \mathcal{O}_{X}(-x)$. 
We have a correspondence

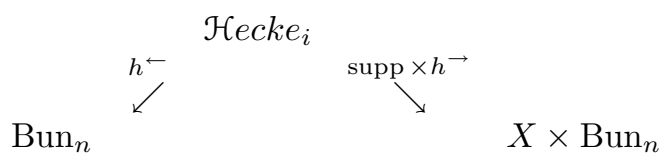

where $h^{\leftarrow}\left(x, \mathcal{M}, \mathcal{M}^{\prime}\right)=\mathcal{M}, h^{\rightarrow}\left(x, \mathcal{M}, \mathcal{M}^{\prime}\right)=\mathcal{M}^{\prime}$, and $\operatorname{supp}\left(x, \mathcal{M}, \mathcal{M}^{\prime}\right)=x$. We will use the same notation for the corresponding maps between the sets of $\mathbb{F}_{q}-$ points.

Let $\mathcal{H e c k e}_{i, x}=\operatorname{supp}^{-1}(x)$. This is a correspondence in $\operatorname{Bun}_{n} \times \operatorname{Bun}_{n}$. Therefore it defines an operator on the space of functions on $G L_{n}(F) \backslash G L_{n}(\mathbb{A}) / G L_{n}(\mathcal{O})$ which takes a function $f$ to the function $h \rightarrow\left(h^{\leftarrow *}(f)\right)$, where $h \rightarrow$ is the operator of integration along the fibers of $h \rightarrow$. It is easy to check that this operator is precisely the $i$ th Hecke operator $H_{i, x}$. Thus, we obtain an interpretation of the generators of the spherical Hecke algebra $\mathcal{H}_{x}$ in terms of Hecke correspondences.

A general cuspidal automorphic representation $\pi$ is unramified away from a finite set of points $S \subset|X|$, but for each $x \in S$ there exists a compact subgroup $K_{x}$ such that the space of $K_{x}$-invariants in $\pi_{x}$ is non-zero. Without loss of generality we may assume that $K_{x}$ is the congruence subgroup of $G L_{n}\left(\mathcal{O}_{x}\right)$ whose elements are congruent to the identity modulo the $m_{x}$ th power of the maximal ideal of $\mathcal{O}_{x}$. Consider the divisor $N=\sum_{x \in S} m_{x}[x]$ on $X$. Denote by $\mathcal{A}_{n, N}$ the subset of $\mathcal{A}_{n}$, which consists of the equivalence classes of those representations $\pi$ which have a non-zero space of invariants with respect to the compact subgroup

$$
K_{N}=\prod_{x \in S} K_{x} \times \prod_{x \in|X|-S} G L_{n}\left(\mathcal{O}_{x}\right) .
$$

If $\pi \in \mathcal{A}_{n, N}$, then the space $\pi_{N}$ of $K_{N}$-invariants of $\pi$ embeds into the space of functions on the double quotient $G L_{n}(F) \backslash G L_{n}(\mathbb{A}) / K_{N}$.

In a similar fashion to Lemma 3.1 one identifies this double quotient with the set of $\mathbb{F}_{q}$-points of the moduli stack $\operatorname{Bun}_{n, N}$ of rank $n$ vector bundles $\mathcal{M}$ on $X$ together with the level structure at $N$, that is a trivialization of the restriction of $\mathcal{M}$ to $N$, considered as a finite subscheme of $X$. The Hecke algebra $\mathcal{H}_{N}^{n}$ of compactly supported functions on $G L_{n}(\mathbb{A})$ bi-invariant with respect to $K_{N}$ acts on the space of functions on $G L_{n}(F) \backslash G L_{n}(\mathbb{A}) / K_{N}$ preserving its subspace $\pi^{K_{N}}$. This action may also be described in terms of Hecke correspondences in $\operatorname{Bun}_{n, N} \times \operatorname{Bun}_{n, N}$ as in the unramified case.

3.3. Deligne's recurrence scheme. The starting point of the proof of the Langlands correspondence is the following recurrence scheme originally suggested by P. Deligne.

Theorem 3.2. Suppose that for each $n^{\prime}=1, \ldots, n-1$ we have a map $\rho_{n^{\prime}}: \mathcal{A}_{n^{\prime}} \rightarrow$ $\mathcal{G}_{n^{\prime}}$ such that for each $\pi \in \mathcal{A}_{n^{\prime}}$ the Frobenius eigenvalues of $\rho_{n^{\prime}}(\pi)$ coincide with the Hecke eigenvalues of $\pi$ at all points where $\pi$ and $\rho_{n^{\prime}}(\pi)$ are unramified. Then there exists a map $\phi_{n}: \mathcal{G}_{n} \rightarrow \mathcal{A}_{n}$ such that for each $\sigma \in \mathcal{G}_{n}$ the Frobenius eigenvalues of $\sigma$ coincide with the Hecke eigenvalues of $\phi_{n}(\sigma)$ at all points where $\sigma$ and $\phi_{n}(\sigma)$ are unramified.

The proof is based on several deep results: Grothendieck's functional equation for the $L$-functions associated to $\ell$-adic Galois representations $[\mathrm{Gr}$, Laumon's product formula for the $\epsilon$-constant appearing in this functional equation La1, and the 
"converse theorems" of Hecke, Weil and Piatetski-Shapiro [PS1, [CPS]. We refer the reader to [La1] and [Laf2] for more details.

The Langlands correspondence is known for $n=1$ by the abelian class field theory. Therefore, in view of Theorem 3.2, in order to establish the Langlands correspondence for all $n>1$ it is sufficient to construct maps $\rho_{n}: \mathcal{A}_{n} \rightarrow \mathcal{G}_{n}$ satisfying the conditions of Theorem 3.2 for all $n>1$. How can this be achieved?

A naive idea is to construct a natural representation of $G L_{n}(\mathbb{A}) \times G_{F}$ defined over $\mathbb{Q}_{\ell}$ which decomposes (over $\overline{\mathbb{Q}}_{\ell}$ ) into a direct sum

$$
\bigoplus_{\pi \in \mathcal{A}_{n}} \pi \otimes \sigma_{\pi}
$$

where each $\sigma_{\pi}$ is an irreducible $n$-dimensional $\ell$-adic representation of $G_{F}$. Then if the Hecke eigenvalues of $\pi$ and Frobenius eigenvalues of $\sigma_{\pi}$ coincide, we can construct the map $\rho_{n}$ by the formula $\pi \mapsto \sigma_{\pi}$. Unfortunately, such a representation of $G L_{n}(\mathbb{A}) \times G_{F}$ does not exist (this is explained in $\mathrm{Kaz}$ ). Instead, Drinfeld proposed to construct a representation of the product $G L_{n}(\mathbb{A}) \times G_{F} \times G_{F}$ which decomposes as

$$
\bigoplus_{\pi \in \mathcal{A}_{n}} \pi \otimes \sigma_{\pi} \otimes \sigma_{\pi}^{\vee},
$$

where $\sigma_{\pi}^{\vee}$ denotes the representation contragredient to $\sigma_{\pi}$ (here and below we will be ignoring the Tate twists).

Drinfeld (for $n=2$ ) and Lafforgue (for $n>2$ ) have constructed such a representation in the "essential" part of the direct limit of the middle $\ell$-adic cohomologies of the moduli spaces of shtukas with level structures. In the next section we will introduce these moduli spaces.

3.4. Moduli spaces of shtukas. As is well-known by now, "shtuka" is a Russian word that may be loosely translated as a "widget". This term was used by Drinfeld for the following objects that he introduced.

First note that the Frobenius endomorphism Fr of $\mathbb{F}_{q}$ defined by the formula $y \mapsto y^{q}$ induces a map $\operatorname{Id} \times \operatorname{Fr}: \bar{X} \rightarrow \bar{X}$, where $\bar{X}=X \underset{\operatorname{Spec} \mathbb{F}_{q}}{\times} \operatorname{Spec} \overline{\mathbb{F}}_{q}$. Given a vector bundle $\mathcal{E}$ on $\bar{X}$, we denote by ${ }^{\tau} \mathcal{E}$ the vector bundle $(\operatorname{Id} \times \operatorname{Fr})^{*}(\mathcal{E})$.

Recall that the skyscraper sheaf supported at $z \in \bar{X}$ is the coherent sheaf whose stalk at $y \in \bar{X}$ is one-dimensional if $y=z$ and is equal to 0 if $y \neq z$.

Definition 3.3. A shtuka of rank $n$ on $\bar{X}$ is a vector bundle $\mathcal{E}$ of rank $n$ on $\bar{X}$ together with a diagram

$$
\mathcal{E} \stackrel{j}{\hookrightarrow} \mathcal{E}^{\prime} \stackrel{t}{\longleftarrow} \tau \mathcal{E}
$$

where $\mathcal{E}^{\prime}$ is another rank $n$ vector bundle on $\bar{X}$ and $t, j$ are injections of the corresponding sheaves of sections such that their cokernels are the skyscraper sheaves supported at the points $0, \infty \in \bar{X}$, called the zero and the pole of the shtuka, respectively.

Note that a vector bundle $\mathcal{E}$ on $\bar{X}$ equipped with an isomorphism ${ }^{\tau} \mathcal{E} \simeq \mathcal{E}$ is the same thing as a vector bundle on $X$. So a shtuka is a mild generalization of the notion of vector bundle on $X$. Indeed, for any shtuka the bundles ${ }^{\tau} \mathcal{E}$ and $\mathcal{E}$ are isomorphic over $\bar{X}-\{0, \infty\}$ and they differ in the simplest possible way at the points 0 and $\infty$. 
The reader may find more information on shtukas and closely related objects, Drinfeld modules, including explicit examples, in [Go].

Let $N=\operatorname{Spec} \mathcal{O}_{N}$ be a finite subscheme of $X$ (equivalently, a divisor on $X$ ). A shtuka with level structure $N$ is a shtuka $\widetilde{\mathcal{E}}=\left(\mathcal{E}, \mathcal{E}^{\prime}, j, t\right)$ such that the points 0 and $\infty$ avoid $N$, together with a trivialization of the restriction of $\mathcal{E}$ to $N$, i.e., an isomorphism $\mathcal{E} \underset{\mathcal{O}_{\bar{X}}}{\otimes \mathcal{O}_{\bar{N}}} \simeq \mathcal{O}_{\bar{N}}^{\oplus n}$ so that the induced trivialization of the restriction of ${ }^{\tau} \mathcal{E}$ to $N$ is compatible with the isomorphism

$$
t \circ j:\left.\left.\mathcal{E}\right|_{\bar{X} \backslash\{0, \infty\}} \stackrel{\sim}{\rightarrow} \mathcal{L}\right|_{\bar{X} \backslash\{0, \infty\}} .
$$

One defines similarly shtukas over $X \times S$, where $S$ is any scheme over $\mathbb{F}_{q}$. This enables one to define an algebraic stack $\mathrm{Cht}_{N}^{n}$ classifying shtukas of rank $n$ with level structure at $N$ on $X$. Drinfeld proved that this is a smooth Deligne-Mumford stack, which means, roughly, that locally it looks like the quotient of a smooth algebraic variety by the action of a finite group. It is equipped with a natural morphism to $(X-N) \times(X-N)$ (taking the pole and zero of a shtuka) of relative dimension $2 n-2$. It carries the action of the "partial" Frobenius endomorphisms Frob $b_{0}$ and Frob $_{\infty}$ corresponding to the zero and pole of the shtuka. In the same way as in Section 3.1 one defines Hecke correspondences in $\mathrm{Cht}_{N}^{n} \underset{(|X|-N)^{2}}{\times} \mathrm{Cht}_{N}^{n}$ which realize the Hecke algebra $\mathcal{H}_{N}^{n}$ of compactly supported functions on $G L_{n}(\mathbb{A})$ bi-invariant with respect to $K_{N}$.

In addition, one has a natural action on $\mathrm{Cht}_{N}^{n}$ of the Picard group of line bundles on $X$ (by tensoring with $\mathcal{E}$ and $\mathcal{E}^{\prime}$ ). Lemma 3.1 implies that the Picard group is isomorphic to the quotient $F^{\times} \backslash \mathbb{A}^{\times} / \mathcal{O}^{\times}$. Let us pick an element $a$ of degree 1 in $\mathbb{A}^{\times}$. Lafforgue denotes the quotient of $\mathrm{Cht}_{N}^{n}$ by the action of the cyclic group generated by the corresponding line bundle by $\mathrm{Cht}_{N}^{n} / a^{\mathbb{Z}}$. This algebraic stack still carries the above actions of Frob $_{0}$, Frob $_{\infty}$ and $\mathcal{H}_{N}^{n}$.

3.5. Strategy of the proof. Let $q^{\prime}, q^{\prime \prime}$ be the two projections $X^{2} \rightarrow X$. For each representation $\sigma$ of $G_{F}$ we obtain by pull-back two representations, $q^{\prime *}(\sigma)$ and $q^{\prime \prime *}(\sigma)$, of the Galois group $G_{\widetilde{F}}$ of the field $\widetilde{F}$ of functions on $X^{2}$. Consider the $\ell$-adic cohomology with compact support of $\mathrm{Cht}_{N}^{n} / a^{\mathbb{Z}}$ over the generic point of $X \times X$. This is naturally a representation of the Hecke algebra $\mathcal{H}_{N}^{n}$ and of $G_{\widetilde{F}}$, whose actions commute with each other. We would like to isolate in this cohomology a subspace that decomposes as

$$
\bigoplus_{\pi \in \mathcal{A}_{n, N}^{a}} \pi_{N} \otimes q^{\prime *}\left(\sigma_{\pi}\right) \otimes q^{\prime \prime *}\left(\sigma_{\pi}^{\vee}\right)
$$

where $\pi_{N}$ is the space of $K_{N}$-invariants in $\pi$, each $\sigma_{\pi}$ is an irreducible $n$-dimensional $\ell$-adic representation of $G_{F}$ unramified in $|X|-N$, and $\mathcal{A}_{n, N}^{a}$ is a subset of $\mathcal{A}_{n, N}$, which consists of those representations on which $a \in G L_{n}(\mathbb{A})$ acts as the identity. If we could show that the Hecke eigenvalues of each $\pi$ in the above formula coincide with the Frobenius eigenvalues of $\sigma_{\pi}$, then in view of the discussion at the end of Section [3.3, this would prove the Langlands correspondence.

Suppose we could isolate this subspace in the cohomology. Then we would need to compute the traces of the operators of the form $f \times\left(\text { Frob }_{0}\right)^{s} \times\left(\text { Frob }_{\infty}\right)^{s}$ on this subspace, where $f \in \mathcal{H}_{N}^{n}$ and $0, \infty \in|X-N|$. If we could establish that these 
traces are equal to

$$
\begin{aligned}
& \sum_{\pi \in \mathcal{A}_{n, N}^{a}} \operatorname{Tr}_{\pi_{N}}(f)\left(z_{1}\left(\pi_{\infty}\right)^{-s / \operatorname{deg}(\infty)}+\ldots+z_{n}\left(\pi_{\infty}\right)^{-s / \operatorname{deg}(\infty)}\right) \times \\
&\left(z_{1}\left(\pi_{0}\right)^{s / \operatorname{deg}(0)}+\ldots+z_{n}\left(\pi_{0}\right)^{s / \operatorname{deg}(0)}\right),
\end{aligned}
$$

then it would not be difficult to prove that our space is indeed isomorphic to (3.1) with all the required compatibilities. But how could we possibly identify the trace over the cohomology with the sum (3.2)?

First of all, we need to apply the Grothendieck-Lefschetz fixed point formula. It expresses the alternating sum of the traces of correspondences over $\ell$-adic cohomologies of a smooth variety as the number of the fixed points of these correspondences. Suppose we could apply this formula to the correspondences $f \times\left(\text { Frob }_{0}\right)^{s} \times\left(\text { Frob }_{\infty}\right)^{s}$ acting on $\mathrm{Cht}_{N}^{n} / a^{\mathbb{Z}}$. We should then compare the number of fixed points appearing in this formula with the Arthur-Selberg trace formula. This formula describes the traces of the operators like $f \in \mathcal{H}_{N}^{n}$ on the space $\mathcal{C}_{\text {cusp }}=\bigoplus_{\pi \in \mathcal{A}_{n}} \pi$ of cuspidal automorphic functions in terms of orbital integrals in $G L_{n}(\mathbb{A})$. We may hope to relate these orbital integrals to the numbers of fixed points in the moduli spaces of shtukas. That would give us the desired expression for the trace of our correspondences acting on the $\ell$-adic cohomology as the sum (3.2).

3.6. From a dream to reality. This is the general strategy of Drinfeld and Lafforgue. Unfortunately, literally it cannot work, because $\mathrm{Cht}_{N}^{n} / a^{\mathbb{Z}}$ is not (quasi)compact: it cannot be covered by finitely many open subsets of finite type. For this reason its cohomology is infinite-dimensional and there are infinitely many fixed points, and so one cannot apply the Grothendieck-Lefschetz formula. To remedy this, Lafforgue introduces open substacks $\mathrm{Cht}_{N}^{n, p} / a^{\mathbb{Z}}$ of finite type in $\mathrm{Cht}_{N}^{n, p} / a^{\mathbb{Z}}$. They are labeled by "Harder-Narasimhan polygons" $p:[0, n] \rightarrow \mathbb{R}_{+}$. Furthermore, using the Arthur-Selberg trace formula (see [Laf1]) Lafforgue computes the numbers of fixed points of the correspondences $f \times\left(\text { Frob }_{0}\right)^{s} \times\left(\text { Frob }_{\infty}\right)^{s}$ in the set of $\mathbb{F}_{q^{-}}$ points of $\mathrm{Cht}_{N}^{n, p} / \mathrm{a}^{\mathbb{Z}}$. The answer is the expression (3.2) plus the the sum of terms that correspond to representations of $G L_{n}(\mathbb{A})$ induced from parabolic subgroups (i.e., those which are not cuspidal).

The problem, however, is that (except for the case when it is equal to the identity) the correspondence $f$ does not stabilize the open subset $\mathrm{Cht}_{N}^{n, p} / a^{\mathbb{Z}}$. Therefore we cannot interpret the number of fixed points as the trace over the cohomology of $\mathrm{Cht}_{N}^{n, p} / a^{\mathbb{Z}}$. To fix this problem, Lafforgue (and Drinfeld for $n=2$ ) introduced compactifications $\overline{\mathrm{Cht}_{N}^{n, p}} / a^{\mathbb{Z}}$ of $\mathrm{Cht}_{N}^{n, p} / a^{\mathbb{Z}}$ by allowing certain degenerations of shtukas. The Hecke correspondences can now be extended to $\overline{\mathrm{Cht}_{N}^{n, p}} / a^{\mathbb{Z}}$. Unfortunately, the compactifications $\overline{\mathrm{Cht}_{N}^{n, p}} / a^{\mathbb{Z}}$ are singular (unless $N=\emptyset$ ). Therefore a priori these Hecke correspondences do not induce linear operators on the cohomologies of $\overline{\operatorname{Cht}_{N}^{n, p}} / a^{\mathbb{Z}}$.

In the case when $n=2$, Drinfeld has shown that $\overline{\mathrm{Cht}_{N}^{2, p}} / a^{\mathbb{Z}}$ is quasi-smooth; i.e., its cohomology exhibits Poincaré duality. Therefore the Hecke correspondences act on the cohomology of $\overline{\mathrm{Cht}_{N}^{2, p}} / a^{\mathbb{Z}}$, and Drinfeld was able to relate their traces to the numbers of fixed points. This allowed him to prove that the cuspidal part of the middle (second in this case) cohomology of $\overline{\mathrm{Cht}_{N}^{2, p}} / a^{\mathbb{Z}}$ indeed decomposes according to formula (3.1) and hence realizes the Langlands correspondence for $G L_{2}$. 
For $n>2$ the stacks $\overline{\mathrm{Cht}_{N}^{n, p}} / a^{\mathbb{Z}}$ are no longer quasi-smooth in general. To deal with this problem Lafforgue introduced another open subset ${\overline{\operatorname{Cht}_{N}^{n, p^{\prime}}}}^{\prime} a^{\mathbb{Z}}$ of $\overline{\mathrm{Cht}_{N}^{n, p}} / a^{\mathbb{Z}}$ defined by the condition that the degenerations of the shtuka avoid $N$. The stack $\overline{\mathrm{Cht}_{N}^{n, p^{\prime}}} / a^{\mathbb{Z}}$ is smooth, and its complement in $\overline{\mathrm{Cht}_{N}^{n, p}} / a^{\mathbb{Z}}$ is a divisor with normal crossings. Moreover, it turns out that the (normalized) Hecke correspondences defined in $\overline{\operatorname{Cht}_{N}^{n, p^{\prime}}} / a^{\mathbb{Z}}$ stabilize $\overline{\operatorname{Cht}_{N}^{n, p}} / a^{\mathbb{Z}}$.

Thus, Lafforgue ended up with three different objects, each with some "good" and "bad" properties. Indeed, $\mathrm{Cht}_{N}^{n} / a^{\mathbb{Z}}$ carries an action of the Hecke algebra by correspondences, but is not of finite type. The stacks $\mathrm{Cht}_{N}^{n, p} / \mathrm{a}^{\mathbb{Z}}$ are of finite type, and it is possible to compute the numbers of fixed points of the Hecke correspondences there, but the correspondences themselves do not preserve them. Finally, on $\overline{\mathrm{Cht}}_{N}^{n, p^{\prime}} / a^{\mathbb{Z}}$ one can write down the Grothendieck-Lefschetz formula for the Hecke correspondences, but it is not clear how to compute either side of this formula.

Lafforgue's ingenious trick is to separate inside the cohomology with compact support of all three objects the "essential" part and the "negligible" part and to show that the "essential" part is the same for all of them. Namely, the negligible part consists of those representations of $G_{\widetilde{F}}$ which appear as direct factors in the tensor products of the form $q^{\prime *}\left(\sigma^{\prime}\right) \otimes q^{\prime \prime *}\left(\sigma^{\prime \prime}\right)$, where $\sigma^{\prime}$ and $\sigma^{\prime \prime}$ are $G_{F}$ representations of dimension less than $n$. The rest is the essential part.

In order to compute this essential part, Lafforgue needed to overcome some formidable technical difficulties. The original Grothendieck-Lefschetz formula is applicable when we have a smooth proper algebraic variety. Here $\overline{\operatorname{Cht}}_{N}^{n, p} / a^{\mathbb{Z}}$ is not a variety but an algebraic stack which is not even proper. Building upon earlier works of Pink and Fujiwara on the Deligne conjecture, Lafforgue proved a new version of the Grothendieck-Lefschetz formula which enabled him to express the trace of a Hecke correspondence and a power of the Frobenius endomorphism on the cohomology of $\overline{\mathrm{Cht}_{N}^{n, p^{\prime}}} / a^{\mathbb{Z}}$ as the number of fixed points in $\mathrm{Cht}_{N}^{n, p} / a^{\mathbb{Z}}$ plus a sum of terms corresponding to various boundary strata in the complement of $\mathrm{Cht}_{N}^{n, p} / a^{\mathbb{Z}}$. By a complicated recurrence argument on $n$ he showed that the latter are all negligible. Therefore he was able to identify the traces of the Hecke operators and the Frobenius endomorphisms acting on the essential part of the middle cohomology with compact support of $\mathrm{Cht}_{N}^{n, p} / a^{\mathbb{Z}}$ (which coincides with that of $\overline{\mathrm{Cht}}_{N}^{n, p^{\prime}} / a^{\mathbb{Z}}$ ) with formula (3.2). This completed his proof of the Langlands correspondence for $G L_{n}$.

In addition, he proved Theorem 2.3 because the Frobenius eigenvalues of irreducible representations of $G_{F}$ are now realized as traces of Frobenius endomorphisms acting on $\ell$-adic cohomology, so that one can apply Deligne's results on Weil's conjectures [De1, De2].

\section{The geometric Langlands Conjecture}

The geometric reformulation of the Langlands conjecture allows one to state it for curves defined over an arbitrary field, not just a finite field. For instance, it may be stated for complex curves, and in this setting one can apply methods of complex algebraic geometry which are unavailable over finite fields. Hopefully, this will eventually help us understand better the general underlying patterns of the 
Langlands correspondence. In this section we will formulate the geometric Langlands conjecture for $G L_{n}$ and discuss briefly its recent proof due to D. Gaitsgory, K. Vilonen and the author.

4.1. Galois representations as local systems. What needs to be done to reformulate the Langlands conjecture geometrically?

As we indicated in Section 2.1 the Galois group $G_{F}$ should be viewed as a kind of fundamental group, and so its $\ell$-adic representations unramified away from a divisor $N$ should be viewed as local systems on $X-N$. This is indeed possible if one defines local systems in terms of $\ell$-adic sheaves.

Let us discuss them briefly. Let $V$ be an algebraic variety over $\mathbb{F}_{q}$, and $\ell$ a prime which does not divide $q$. Then one defines the category of $\ell$-adic sheaves on $V$. The construction involves several steps (see, e.g., [Mi], [FK]). First one considers locally constant $\mathbb{Z} / \ell^{m} \mathbb{Z}$-sheaves on $V$ in the étale topology (in which the role of open subsets is played by étale morphisms $U \rightarrow V)$. A $\mathbb{Z}_{\ell}$-sheaf on $V$ is by definition a system $\left(\mathcal{F}_{m}\right)$ of locally constant $\mathbb{Z} / \ell^{m} \mathbb{Z}$-sheaves satisfying natural compatibilities. Then one defines the category of $\mathbb{Q}_{\ell}$-sheaves by killing the torsion sheaves in the category of $\mathbb{Z}_{\ell}$-sheaves. In a similar fashion one defines the category of $E$-sheaves on $V$, where $E$ is a finite extension of $\mathbb{Q}_{\ell}$. Finally, one takes the direct limit of the categories of $E$-sheaves on $X$, and the objects of this category are called the lisse $\ell$-adic sheaves on $V$, or $\ell$-adic local systems. An $\ell$-adic local system on $X-N$ of rank $n$ is the same as an $n$-dimensional $\ell$-adic representation of $G_{F}$ unramified everywhere on $X-N$.

Thus, we can now interpret Galois representations geometrically: these are $\ell$-adic local systems on $X$. The notion of local systems makes sense if $X$ is defined over other fields. For example, if $X$ is a smooth projective curve over $\mathbb{C}$, a local system is a vector bundle on $X$ with a flat connection, or equivalently, a homomorphism from the fundamental group $\pi_{1}(X)$ to $G L_{n}(\mathbb{C})$.

4.2. From functions to sheaves. Next, we wish to interpret geometrically automorphic representations. Let us restrict ourselves to unramified representations. As we explained in Section 3.1, one can attach to such a representation $\pi$ a nonzero function $f_{\pi}$ (unique up to a scalar) on $G L_{n}(F) \backslash G L_{n}(\mathbb{A}) / G L_{n}(\mathcal{O})$, which is an eigenfunction of the Hecke algebras $\mathcal{H}_{x}, x \in|X|$. In fact, this function completely determines the representation $\pi$, so instead of considering the set of equivalence classes of unramified cuspidal representations of $G L_{n}(\mathbb{A})$, one may consider the set of unramified automorphic functions associated to them (defined up to a scalar).

The key step in the geometric reformulation of this notion is the Grothendieck fonctions-faisceaux dictionary. Let $V$ be an algebraic variety over $\mathbb{F}_{q}$. One generalizes the above definition of an $\ell$-adic local system on $V$ by allowing the $\mathbb{Z} / \ell^{n} \mathbb{Z}-$ sheaves $\mathcal{F}_{n}$ to be constructible, i.e., for which there exists a stratification of $V$ by locally closed subvarieties $V_{i}$ such that the sheaves $\left.\mathcal{F}\right|_{V_{i}}$ are locally constant. As a result, one obtains the notion of a constructible $\ell$-adic sheaf on $V$, or an $\ell$-adic sheaf, for brevity. Let $\mathcal{F}$ be such a sheaf and $x$ be an $\mathbb{F}_{q_{1}}$-point of $V$, where $q_{1}=q^{m}$. Then one has the Frobenius conjugacy class $\operatorname{Fr}_{x}$ acting on the stalk $\mathcal{F}_{x}$ of $\mathcal{F}$ at $x$. Hence we can define a function $\mathbf{f}_{q_{1}}(\mathcal{F})$ on the set of $\mathbb{F}_{q_{1}}$-points of $V$, whose value at $x$ is $\operatorname{Tr}\left(\operatorname{Fr}_{x}, \mathcal{F}_{x}\right)$. 
More generally, if $\mathcal{K}$ is a complex of $\ell$-adic sheaves, one defines a function $\mathrm{f}_{q_{1}}(\mathcal{K})$ on $V\left(\mathbb{F}_{q_{1}}\right)$ by taking the alternating sums of the traces of $\mathrm{Fr}_{x}$ on the stalk cohomologies of $\mathcal{K}$ at $x$. The map $\mathcal{K} \rightarrow \mathbf{f}_{q_{1}}(\mathcal{K})$ intertwines the natural operations on sheaves with natural operations on functions (see [La1], Sect. 1.2). For example, pull-back of a sheaf corresponds to the pull-back of a function, and push-forward of a sheaf with compact support corresponds to the fiberwise integration of a function (this follows from the Grothendieck-Lefschetz trace formula).

We wish to identify a natural abelian category in the derived category of $\ell$-adic sheaves such that the map $\mathcal{K} \mapsto\left(\mathbf{f}_{q_{1}}(\mathcal{K})\right)_{q_{1}=q^{m}}$ is injective. The naive category of $\ell$-adic sheaves is not a good choice for various reasons; for instance, it is not stable under the Verdier duality. The correct choice is the abelian category of perverse sheaves. These are complexes of $\ell$-adic sheaves on $V$ satisfying certain restrictions on the degrees of their non-zero stalk cohomologies (see [BBD]). Examples are $\ell$-adic local systems on a smooth variety $V$, placed in the cohomological degree equal to $-\operatorname{dim} V$. Unlike ordinary sheaves, the perverse sheaves have the following remarkable property: an irreducible perverse sheaf on a variety $V$ is completely determined by its restriction to an arbitrary open dense subset, provided that this restriction is non-zero.

Optimistically, one can hope that all "interesting" functions on $V\left(\mathbb{F}_{q_{1}}\right)$ come from perverse sheaves by taking the traces of the Frobeniuses (or is it Frobenii?). Unramified automorphic functions on $G L_{n}(F) \backslash G L_{n}(\mathbb{A}) / G L_{n}(\mathcal{O})$ are certainly "interesting". Therefore one hopes that they all come from perverse sheaves on the moduli stack $\operatorname{Bun}_{n}$, whose set of points is $G L_{n}(F) \backslash G L_{n}(\mathbb{A}) / G L_{n}(\mathcal{O})$, according to Lemma 3.1

Thus, we have identified the geometric objects which should replace unramified automorphic functions: these are perverse sheaves on $\mathrm{Bun}_{n}$. This concept also makes sense if $X$ is defined over other fields, for example, the field of complex numbers (see, e.g., GM]).

4.3. Hecke eigensheaves and the geometric Langlands conjecture. But how to formulate the Hecke eigenfunction condition which unramified automorphic functions satisfy in sheaf-theoretic terms? The key is the description of the Hecke operators in terms of the Hecke correspondences that was explained in Section 3.1 We use these correspondences to define the Hecke functors $\mathrm{H}_{i}$ from the category of perverse sheaves on $\operatorname{Bun}_{n}$ to the derived category of sheaves on $X \times \operatorname{Bun}_{n}$ by the formula (here and below we will ignore cohomological shifts and Tate twists)

$$
\mathrm{H}_{i}(\mathcal{K})=\left(\operatorname{supp} \times h^{\rightarrow}\right) ! h^{\leftarrow *}(\mathcal{K}) .
$$

Definition 4.1. Consider an $\ell-$ adic local system $E$ of rank $n$ on $X$. A perverse sheaf $\mathcal{K}$ on $\operatorname{Bun}_{n}$ is called a Hecke eigensheaf with respect to $E$ if $\mathcal{K} \neq 0$ and we are given isomorphisms

$$
\mathrm{H}_{n}^{i}(\mathcal{K}) \simeq \wedge^{i} E \otimes \mathcal{K}, \quad i=1, \ldots, n,
$$

where $\wedge^{i} E$ is the ith exterior power of $E$.

Let $\sigma$ be an $n$-dimensional unramified $\ell$-adic representation of $G_{F}$ and $E_{\sigma}$ the corresponding $\ell$-adic local system on $X$. Then

$$
\operatorname{Tr}\left(\operatorname{Fr}_{x}, E_{x}\right)=\operatorname{Tr}\left(\sigma\left(\operatorname{Fr}_{x}\right), \overline{\mathbb{Q}}_{\ell}^{n}\right)=\sum_{i=1}^{n} z_{i}\left(\sigma_{x}\right)
$$


(see Section 2.1 for the definition of $z_{i}\left(\sigma_{x}\right)$ ), and so

$$
\operatorname{Tr}\left(\operatorname{Fr}_{x}, \wedge^{i} E_{x}\right)=s_{i}\left(z_{1}\left(\sigma_{x}\right), \ldots, z_{n}\left(\sigma_{x}\right)\right)
$$

where $s_{i}$ is the $i$ th elementary symmetric polynomial. Therefore we find that the function $\mathrm{f}_{q}(\mathcal{K})$ on $G L_{n}(F) \backslash G L_{n}(\mathbb{A}) / G L_{n}(\mathcal{O})$ associated to a Hecke eigensheaf $\mathcal{K}$ satisfies

$$
H_{i, x} \cdot \mathbf{f}_{q}(\mathcal{K})=s_{i}\left(z_{1}\left(\sigma_{x}\right), \ldots, z_{n}\left(\sigma_{x}\right)\right) \mathbf{f}_{q}(\mathcal{K})
$$

(up to some $q$-factors). In other words, $\mathrm{f}_{q}(\mathcal{K})$ is a Hecke eigenfunction whose Hecke eigenvalues are equal to the Frobenius eigenvalues of $\sigma$. Hence we are naturally led to the following geometric Langlands conjecture, which is due to Drinfeld and Laumon La2].

The statement of this conjecture was proved by Deligne for $G L_{1}$ (we recall it in the next section) and by Drinfeld in the case of $G L_{2}$ [Dr4. These works motivated the conjecture in the case of $G L_{n}$, which has recently been proved in [FGV], Ga]. So we now have the following result, in which $X$ is a smooth projective connected curve defined either over a finite field or over the field of complex numbers. As before, we denote by $\operatorname{Bun}_{n}$ the moduli stack of rank $n$ bundles on $X$. It is a disjoint union of connected components $\operatorname{Bun}_{n}^{d}$ corresponding to vector bundles of degree $d$.

Theorem 4.2. For each irreducible rank $n$ local system $E$ on $X$ there exists a perverse sheaf $\mathrm{Aut}_{E}$ on $\mathrm{Bun}_{n}$, irreducible on each connected component $\mathrm{Bun}_{n}^{d}$, which is a Hecke eigensheaf with respect to $E$.

4.4. Geometric abelian class field theory. Let us consider Deligne's proof of the $n=1$ case of Theorem 4.2 (see [La2]). In this case $B u n_{n}^{d}$ is essentially the component $\mathrm{Pic}_{d}$ of the Picard variety Pic of $X$ classifying the line bundles on $X$ of degree $d$. In order to prove Conjecture 4.2 in this case, we need to assign to each rank one local system $E$ on $X$ a perverse sheaf $A u_{E}$ on Pic which satisfies the following Hecke eigensheaf property:

$$
h^{\leftarrow *}\left(\mathrm{Aut}_{E}\right) \simeq E \otimes \mathrm{Aut}_{E},
$$

where $h^{\leftarrow}: X \times$ Pic $\rightarrow$ Pic is given by $(\mathcal{L}, x) \mapsto \mathcal{L}(x)$.

Consider the Abel-Jacobi map $\pi_{d}: S^{d} X \rightarrow$ Pic $_{d}$ sending the divisor $D$ to the line bundle $\mathcal{O}(D)$. If $d>2 g-2$, then $\pi_{d}$ is a projective bundle, with the fibers $\pi_{d}^{-1}(\mathcal{L})=\mathbb{P} H^{0}(X, \mathcal{L})$. It is easy to construct a local system $E^{(d)}$ on $\bigcup_{d>2 g-2} S^{d} X$ satisfying an analogue of the Hecke eigensheaf property

$$
\widetilde{h}^{\leftarrow *}\left(E^{(d)}\right) \simeq E \otimes E^{(d)},
$$

where $\widetilde{h}^{\leftarrow}: S^{d} X \times X \rightarrow S^{d+1} X$ is given by $(D, x) \mapsto D+[x]$. Namely, let

$$
\operatorname{sym}^{d}: X^{n} \rightarrow S^{n} X
$$

be the symmetrization map and set $E^{(d)}=\left(\operatorname{sym}_{*}^{d}\left(E^{\bigotimes n}\right)\right)^{S_{d}}$.

So we have local systems $E^{(d)}$ on $S^{d} X, d>2 g-2$, which form a Hecke eigensheaf, and we need to prove that they descend to $\mathrm{Pic}_{d}$ under the Abel-Jacobi map $\pi_{d}$. In other words, we need to prove that the restriction of $E^{(d)}$ to each fiber of $\pi_{d}$ is a constant sheaf. Since $E^{(d)}$ is a local system, these restrictions are locally constant. But the fibers of $\pi_{d}$ are projective spaces, hence simply-connected. Therefore any locally constant sheaf along the fiber is constant! So there exists a local system $\mathrm{Aut}_{E}^{d}$ on $\mathrm{Pic}_{d}$ such that $E^{(d)}=\pi_{d}^{*}\left(\mathrm{Aut}_{E}^{d}\right)$. Formula (4.4) implies that the sheaves 
$\mathrm{Aut}_{E}^{d}$ form a Hecke eigensheaf on $\bigcup_{d>2 g-2} \mathrm{Pic}_{d}$. We extend them to the remaining components of Pic by using the Hecke eigensheaf property (4.3). Namely, we pick a point $x \in|X|$ and set $\operatorname{Aut}_{E}^{d}=E_{x}^{*} \otimes h_{x}^{\leftarrow *}\left(\right.$ Aut $\left._{E}^{d+1}\right)$, where $h_{x}^{\leftarrow}(\mathcal{L})=\mathcal{L}(x)$. Then the fact that the restrictions of $E^{(d)}$ to the fibers of $\pi_{d}$ is constant implies that the resulting sheaves Aut ${ }_{E}^{d}$ for $d \leq 2 g-2$ do not depend on the choice of $x$. Thus, we obtain a Hecke eigensheaf on the entire Pic, and this completes Deligne's proof of the geometric Langlands conjecture for $n=1$.

Let us consider the case when $X$ is defined over a finite field. Then to the sheaf Aut $E$ we attach a function on $F^{\times} \backslash \mathbb{A}^{\times} / \mathcal{O}^{\times}$, which is the set of $\mathbb{F}_{q}$-points of Pic. This function is a Hecke eigenfunction $f_{\sigma}$ with respect to a one-dimensional Galois representation $\sigma$ corresponding to $E$; i.e., it satisfies the equation $f_{\sigma}(\mathcal{L}(x))=$ $\sigma\left(\operatorname{Fr}_{x}\right) f_{\sigma}(\mathcal{L})$ (since $\sigma$ is one-dimensional, we do not need to take the trace). We could try to construct this function proceeding in the same way as above. Namely, we define first a function $f_{\sigma}^{\prime}$ on the set of all divisors on $X$ by the formula

$$
f_{\sigma}^{\prime}\left(\sum_{i} n_{i}\left[x_{i}\right]\right)=\prod_{i} \sigma\left(\operatorname{Fr}_{x_{i}}\right)^{n_{i}}
$$

This function clearly satisfies an analogue of the Hecke eigenfunction condition. It remains to show that the function $f_{\sigma}^{\prime}$ descends to $\operatorname{Pic}\left(\mathbb{F}_{q}\right)$, namely, that if two divisors $D$ and $D^{\prime}$ are rationally equivalent, then $f_{\sigma}^{\prime}(D)=f_{\sigma}^{\prime}\left(D^{\prime}\right)$. This is equivalent to the identity

$$
\prod_{i} \sigma\left(\operatorname{Fr}_{x_{i}}\right)^{n_{i}}=1, \quad \text { if } \quad \sum_{i} n_{i}\left[x_{i}\right]=(g),
$$

where $g$ is an arbitrary rational function on $X$. This identity is a non-trivial reciprocity law which has been proved in the abelian class field theory by Lang and Rosenlicht (see [Se] ).

It is instructive to contrast this to Deligne's geometric proof reproduced above. When we replace functions by sheaves we can use additional information which is "invisible" at the level of functions, such as the fact that the sheaf corresponding to the function $f_{\sigma}^{\prime}$ is locally constant and that the fibers of the Abel-Jacobi map are simply-connected. This is one of the main motivations for studying the Langlands correspondence in the geometric setting.

4.5. The idea of the proof for general $n$. Observe that for large $d$ the variety $S^{d} X$ may be interpreted as the moduli space $\operatorname{Bun}_{1}^{\prime}$ of pairs $(\mathcal{L}, s)$, where $\mathcal{L}$ is a line bundle on $X$ and $s$ is its section. We first constructed a Hecke eigensheaf on Bun ${ }_{1}^{\prime}$ and then showed that it descends to Bun ${ }_{1}$. This is the main idea of the construction of $\mathrm{Aut}_{E}$ for general $n$ as well.

At the level of functions this construction is due to Weil [W] and JacquetLanglands JL] for $n=2$, and Shalika Sh] and Piatetski-Shapiro [PS1] for general $n$. They attach to an unramified $n$-dimensional representation $\sigma$ of $G_{F}$ a function $f_{\sigma}^{\prime}$ on the set of isomorphism classes of pairs $(\mathcal{M}, s)$, where $\mathcal{M} \in \operatorname{Bun}_{n}$ is a rank $n$ bundle on $X$ and $s$ is a regular non-zero section of $\mathcal{M}$. Then it remains to show that this function is independent of the section, i.e., descends to the set of isomorphism classes of rank $n$ bundles on $X$, which is the double quotient $G L_{n}(F) \backslash G L_{n}(\mathbb{A}) / G L_{n}(\mathcal{O})$.

We reformulate this geometrically. Let $\operatorname{Bun}_{n}^{\prime}$ be the moduli stack of pairs $(\mathcal{N}, s)$, where $\mathcal{N}$ is a rank $n$ bundle on $X$ and $s$ is a regular non-zero section of $L$. Let 
$E$ be an irreducible rank $n$ local system on $X$. Building on the ideas of Drinfeld's work [Dr4], Laumon gave a conjectural construction of the Hecke eigensheaf Aut $E$ in La2], La3]. More precisely, he attached to each rank $n$ local system $E$ on $X$ a complex of perverse sheaves $\mathrm{Aut}_{E}^{\prime}$ on $\mathrm{Bun}_{n}^{\prime}$ and conjectured that if $E$ is irreducible, then this sheaf descends to a perverse sheaf $\mathrm{Aut}_{E}$ on $\mathrm{Bun}_{n}$ (irreducible on each component), which is a Hecke eigensheaf with respect to $E$.

In the paper [FGKV] it was shown that the function on the set of points $\operatorname{Bun}_{n}^{\prime}\left(\mathbb{F}_{q}\right)$ associated to $\mathrm{Aut}_{E}^{\prime}$ agrees with the function $f_{\sigma}^{\prime}$ constructed previously, as anticipated by Laumon La3]. This provided a consistency check for Laumon's construction. Next, in [FGV], Gaitsgory, Vilonen and I formulated a certain vanishing conjecture and proved that Laumon's construction indeed produces a perverse sheaf $\mathrm{Aut}_{E}$ on $\mathrm{Bun}_{n}$ with the desired properties whenever the vanishing conjecture holds for $E$. In other words, the vanishing conjecture implies the geometric Langlands conjecture for curves over any field. Moreover, in the case when this field is $\mathbb{F}_{q}$, we derived the vanishing conjecture (and hence the geometric Langlands conjecture) from the results of Lafforgue [Laf2]. Finally, Gaitsgory [Ga gave another proof of the vanishing conjecture, valid for curves both over $\mathbb{F}_{q}$ and over $\mathbb{C}$. In the next section we will state this vanishing conjecture.

4.6. The vanishing conjecture. Denote by $\operatorname{Coh}_{n}$ the stack classifying coherent sheaves on $X$ of generic rank $n$, and by $\operatorname{Coh}_{n}^{d}$ its connected component corresponding to coherent sheaves of degree $d$.

In [La2 Laumon associated to an arbitrary local system $E$ of rank $n$ on $X$ a perverse sheaf $\mathcal{L}_{E}$ on $\operatorname{Coh}_{0}$. Let us recall his construction. Denote by $\operatorname{Coh}_{0}^{\text {rss }}$ the open substack of $\mathrm{Coh}_{0}$ corresponding to regular semi-simple torsion sheaves. Thus, a geometric point of $\mathcal{C o h}_{0}$ belongs to $\operatorname{Coh}_{0}^{\text {rss }}$ if the corresponding coherent sheaf on $X$ is a direct sum of skyscraper sheaves of length one supported at distinct points of $X$. Let $\operatorname{Coh}_{0}^{\mathrm{rss}, d}=\operatorname{Coh}_{0}^{\mathrm{rss}} \cap \mathcal{C} o h_{0}^{d}$. We have a natural smooth map $\left(S^{d} X-\Delta\right) \rightarrow \operatorname{Coh}_{0}^{\mathrm{rss}, d}$.

Let $E^{(d)}$ be the $d$ th symmetric power of $E$ defined as in Section 4.4, i.e., $E^{(d)}=$ $\left(\operatorname{sym}_{*}\left(E^{\bigotimes d}\right)\right)^{S_{d}}$, where sym : $X^{d} \rightarrow S^{d} X$. This is a perverse sheaf on $S^{d} X$, though it is not a local system if $n>1$. However, its restriction $\left.E^{(d)}\right|_{S^{d} X-\Delta}$ is a local system, which descends to a local system $\stackrel{\circ}{\mathcal{L}}_{E}^{d}$ on $\operatorname{Coh}_{0}^{\text {rss, } d}$. The perverse sheaf $\mathcal{L}_{E}^{d}$ on $\mathcal{C} o h_{0}^{d}$ is by definition the canonical extension of $\stackrel{\circ}{\mathcal{L}}_{E}^{d}$ to a perverse sheaf on $\operatorname{Coh}_{0}^{d}$ called the Goresky-MacPherson extension. We denote by $\mathcal{L}_{E}$ the perverse sheaf on Coh $h_{0}$ whose restriction to $\operatorname{Coh}_{0}^{d}$ equals $\mathcal{L}_{E}^{d}$.

Using the perverse sheaf $\mathcal{L}_{E}^{d}$, we define the averaging functor $\mathrm{H}_{k, E}^{d}$ on the derived category of perverse sheaves on $\mathrm{Bun}_{k}$, where the positive integer $k$ is independent of $n$, the rank of the local system $E$. For $d \geq 0$, introduce the stack $\operatorname{Mod}_{k}^{d}$, which classifies the data of triples $\left(\mathcal{M}, \mathcal{M}^{\prime}, \beta: \mathcal{M} \hookrightarrow \mathcal{M}^{\prime}\right)$, where $\mathcal{M}, \mathcal{M}^{\prime} \in \operatorname{Bun}_{k}$ and $\beta$ is an embedding of coherent sheaves such that the quotient $\mathcal{M}^{\prime} / \mathcal{M}$ is a torsion sheaf of length $d$. We have the diagram

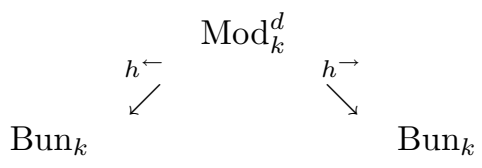


where $h^{\leftarrow}$ (resp., $h^{\rightarrow}$ ) denotes the morphism sending a triple $\left(\mathcal{M}, \mathcal{M}^{\prime}, \beta\right)$ to $\mathcal{M}$ (resp., $\left.\mathcal{M}^{\prime}\right)$. In addition, we have a natural smooth morphism $\pi: \operatorname{Mod}_{k}^{d} \rightarrow \operatorname{Coh}_{0}^{d}$, which sends a triple $\left(\mathcal{M}, \mathcal{M}^{\prime}, \beta\right)$ to the torsion sheaf $\mathcal{M}^{\prime} / \mathcal{M}$.

The averaging functor $\mathrm{H}_{k, E}^{d}$ is defined by the formula

$$
\mathcal{K} \mapsto h_{!}^{\rightarrow}\left(h^{\leftarrow *}(\mathcal{K}) \otimes \pi^{*}\left(\mathcal{L}_{E}^{d}\right)\right) .
$$

The following theorem was stated as a conjecture in [FGV] and proved in [FGV] (when $X$ is defined over a finite field) and in $\mathrm{Ga}$. (when $X$ is defined over a finite field or over $\mathbb{C}$ ).

Theorem 4.3. Let $E$ be an irreducible local system of rank $n$ on $X$. Then for all $k=1, \ldots, n-1$ and all d satisfying $d>k n(2 g-2)$, the functor $\mathrm{H}_{k, E}^{d}$ is identically equal to 0 .

The geometric Langlands conjecture for $G L_{n}$ follows from this theorem together with the main theorem of [FGV], which states that if Theorem 4.3 holds for an irreducible rank $n$ local system $E$, then the geometric Langlands Conjecture 4.2 also holds for $E$.

In the case when $k=1$ Theorem 4.3 has been proved earlier by Deligne, and this result was one of the main steps in Drinfeld's proof of the geometric Langlands conjecture for $G L_{2}$ [Dr4]. In this case the result may be reformulated without using Laumon's sheaf. Recall the Abel-Jacobi map $\pi_{d}: S^{d} X \rightarrow \mathrm{Pic}_{d}$ introduced in Section 4.4. Then for any irreducible rank $n>1$ local system $E$ on $X$ we have

$$
\pi_{d *}\left(E^{(d)}\right)=0, \quad \forall d>n(2 g-2) .
$$

It is not difficult to show that this statement is equivalent to the following result: $H^{\bullet}\left(S^{d} X,\left(E \otimes E^{\prime}\right)^{(d)}\right)=0$ for any rank one local system $E^{\prime}$ on $X$. To prove that, observe that since $E$ is irreducible and has rank $n>1$, so is $E \otimes E^{\prime}$. Therefore we have $H^{0}\left(X, E \otimes E^{\prime}\right)=H^{2}\left(X, E \otimes E^{\prime}\right)=0$. But then by the Künneth formula we have

$$
H^{\bullet}\left(S^{d} X,\left(E \otimes E^{\prime}\right)^{(d)}\right)=\wedge^{d} H^{1}\left(X, E \otimes E^{\prime}\right) .
$$

Note that $\operatorname{dim} H^{1}\left(X, E \otimes E^{\prime}\right)=n(2 g-2)$, which follows immediately from the computation of the Euler characteristic of $H^{\bullet}\left(X, E_{0}\right)$, where $E_{0}$ is the trivial local system of rank $n$. Therefore

$$
H^{\bullet}\left(S^{d} X,\left(E \otimes E^{\prime}\right)^{(d)}\right)=0, \quad \forall d>n(2 g-2)
$$

which is what we needed to prove.

\section{From $G L_{n}$ TO OTHER REDUCTIVE GROUPS}

One adds a new dimension to the Langlands Program by considering arbitrary reductive groups instead of the group $G L_{n}$. This is when some of the most beautiful and mysterious aspects of the program are revealed, such as the appearance of the Langlands dual group. In this section we will trace the appearance of the dual group in the classical context and then talk about its geometrization/categorification. 
5.1. The spherical Hecke algebra for an arbitrary reductive group. Suppose we want to find an analogue of the Langlands correspondence from Theorem [2.2 where instead of automorphic representations of $G L_{n}(\mathbb{A})$ we consider automorphic representations of $G(\mathbb{A})$, where $G$ is a (connected, split) reductive group over $\mathbb{F}_{q}$. We wish to relate those representations to some data corresponding to the Galois group $G_{F}$. In the case of $G L_{n}$ this relation satisfies an important compatibility condition that the Hecke eigenvalues of an automorphic representation coincide with the Frobenius eigenvalues of the corresponding Galois representation. Now we need to find an analogue of this compatibility condition for general reductive groups. The first step is to understand the structure of the spherical Hecke algebra $\mathcal{H}_{x}$. For $G=G L_{n}$ we saw that this algebra is isomorphic to the algebra of symmetric Laurent polynomials in $n$ variables. Now we need to give a similar description of $\mathcal{H}_{x}$ for a general group $G$.

Let $G$ be a connected reductive group over a finite field $\mathbb{F}_{q}$ which is split over $\mathbb{F}_{q}$, i.e., contains a maximal torus $T$ which is isomorphic to a power of the multiplicative group. Then we attach to this torus two lattices, $P$ and $P^{\vee}$, or characters and cocharacters, respectively. They contain subsets $\Delta$ and $\Delta^{\vee}$ of roots and coroots of $G$, respectively (see $\overline{S p}$ for more details). Let us pick a point $x \in|X|$ and assume for simplicity that its residue field is $\mathbb{F}_{q}$. To simplify notation we will omit the index $x$ from our formulas in this section. Thus, we will write $\mathcal{H}, F, \mathcal{O}$ for $\mathcal{H}_{x}, F_{x}, \mathcal{O}_{x}$, etc.

The Hecke algebra $\mathcal{H}=\mathcal{H}(G(F), G(\mathcal{O}))$ is by definition the space of $\overline{\mathbb{Q}}_{\ell}$-valued compactly supported functions on $G(F)$ which are bi-invariant with respect to the maximal compact subgroup $G(\mathcal{O})$. It is equipped with the convolution product

$$
\left(f_{1} \cdot f_{2}\right)(g)=\int_{G} f_{1}(x) f_{2}\left(g x^{-1}\right) d x
$$

where $d x$ is the Haar measure on $G(F)$ normalized so that the volume of $G(\mathcal{O})$ is equal to 1.

We have a natural restriction homomorphism $\mathcal{H} \rightarrow \mathcal{H}(T(F), T(\mathcal{O}))$. The Hecke algebra $\mathcal{H}(T(F), T(\mathcal{O}))$ is easy to describe. For each $\lambda \in P^{\vee}$ we have an element $\lambda(t) \in T(F)$, where $t$ is a uniformizer in $\mathcal{O}$, and $T(\mathcal{O}) \backslash T(F) / T(\mathcal{O})=\{\lambda(t)\}_{\lambda \in P^{\vee}}$. Therefore $\mathcal{H}(T(F), T(\mathcal{O}))$ is the group algebra $\overline{\mathbb{Q}}_{\ell}\left[P^{\vee}\right]$ of $P^{\vee}$. The following result is called the Satake isomorphism.

Theorem 5.1. The homomorphism $\mathcal{H} \rightarrow \mathcal{H}(T(F), T(\mathcal{O}))=\overline{\mathbb{Q}}_{\ell}\left[P^{\vee}\right]$ is injective and its image is equal to the subalgebra $\overline{\mathbb{Q}}_{\ell}\left[P^{\vee}\right]^{W}$ of $W$-invariants, where $W$ is the Weyl group of $G$.

A crucial observation of $\mathrm{R}$. Langlands was that $\overline{\mathbb{Q}}_{\ell}\left[P^{\vee}\right]^{W}$ is nothing but the representation ring of the group ${ }^{L} G\left(\overline{\mathbb{Q}}_{\ell}\right)$, the Langlands dual group of $G$ [L2]. By definition, ${ }^{L} G\left(\overline{\mathbb{Q}}_{\ell}\right)$ is the reductive group over $\overline{\mathbb{Q}}_{\ell}$ with a maximal torus ${ }^{L} T\left(\overline{\mathbb{Q}}_{\ell}\right)$ dual to $T$, so that its lattices of characters and cocharacters are those of $T$ interchanged, and with the sets of roots and coroots being those of $G$ also interchanged (see $[\mathrm{Sp}$ ). For instance, the dual of $G L_{n}$ is again $G L_{n}, S L_{n}$ is dual to $P G L_{n}, S O_{2 n+1}$ is dual to $S p_{n}$, and $S O_{2 n}$ is self-dual.

Let $\operatorname{Rep}{ }^{L} G$ be the Grothendieck ring of the category of finite-dimensional representations of ${ }^{L} G\left(\overline{\mathbb{Q}}_{\ell}\right)$. Then the character homomorphism $\operatorname{Rep}{ }^{L} G \rightarrow \overline{\mathbb{Q}}_{\ell}\left[P^{\vee}\right]$ is injective and its image is equal to $\overline{\mathbb{Q}}_{\ell}\left[P^{\vee}\right]^{W}$. Therefore Theorem $[5.1$ may be interpreted as saying that $\mathcal{H} \simeq \operatorname{Rep}{ }^{L} G$. It follows then that the one-dimensional representations of $\mathcal{H}$ are nothing but the semi-simple conjugacy classes of ${ }^{L} G\left(\overline{\mathbb{Q}}_{\ell}\right)$. 
Indeed, if $\gamma$ is a semi-simple conjugacy class in ${ }^{L} G\left(\overline{\mathbb{Q}}_{\ell}\right)$, then we attach to it a one-dimensional representation of $\mathcal{H}$ by the formula $[V] \mapsto \operatorname{Tr}(\gamma, V)$.

5.2. Towards the Langlands correspondence for an arbitrary reductive group. Now we can formulate for an arbitrary reductive group $G$ an analogue of the compatibility statement in the Langlands conjecture, Conjecture 2.2, for $G L_{n}$. Namely, suppose that $\pi=\bigotimes_{x \in|X|}^{\prime} \pi_{x}$ is a cuspidal automorphic representation of $G(\mathbb{A})$. For all but finitely many $x \in|X|$ the representation $\pi_{x}$ of $G\left(F_{x}\right)$ is unramified; i.e., the space of $G\left(\mathcal{O}_{x}\right)$-invariants in $\pi_{x}$ is non-zero. Then this space of $G\left(\mathcal{O}_{x}\right)$-invariants is one-dimensional and so $\mathcal{H}_{x}$ acts on it via a character, which by Theorem $\left[5.1\right.$ corresponds to a semi-simple conjugacy class $\gamma_{x}$ in ${ }^{L} G\left(\overline{\mathbb{Q}}_{\ell}\right)$. Thus, we attach to an automorphic representation a collection $\left\{\gamma_{x}\right\}$ of semi-simple conjugacy classes in ${ }^{L} G\left(\overline{\mathbb{Q}}_{\ell}\right)$ for almost all points of $X$. Therefore on the other side of the Langlands correspondence we need some sort of Galois data which would also give us such a collection of conjugacy classes.

The candidate that immediately comes to mind is a homomorphism

$$
\sigma: G_{F} \rightarrow{ }^{L} G\left(\overline{\mathbb{Q}}_{\ell}\right)
$$

which is almost everywhere unramified. Then we may attach to $\sigma$ a collection of conjugacy classes $\left\{\sigma\left(\operatorname{Fr}_{x}\right)\right\}$ of ${ }^{L} G\left(\overline{\mathbb{Q}}_{\ell}\right)$ at almost all points of $X$. So in the first approximation we may formulate the Langlands correspondence for general reductive groups as a correspondence between automorphic representations of $G(\mathbb{A})$ and Galois homomorphisms $G_{F} \rightarrow{ }^{L} G\left(\overline{\mathbb{Q}}_{\ell}\right)$ which satisfies the following compatibility condition: if $\pi$ corresponds to $\sigma$, then the ${ }^{L} G$-conjugacy classes attached to $\pi$ through the action of the Hecke algebra are the same as the Frobenius ${ }^{L} G$-conjugacy classes attached to $\sigma$.

Unfortunately, the situation is not as clear-cut as in the case of $G L_{n}$ because many of the results which facilitate the Langlands correspondence for $G L_{n}$ are no longer true in general. For instance, it is not true that the collection of the Hecke conjugacy classes determines the automorphic representation uniquely or that the collection of the Frobenius conjugacy classes determines the Galois representation uniquely in general. Therefore even the statement of the Langlands conjecture becomes a much more subtle issue for a general reductive group. However, the main idea appears to be correct: there is a relationship, still very mysterious, alas, between automorphic representations of $G(\mathbb{A})$ and homomorphisms from the Galois group $G_{F}$ to the Langlands dual group ${ }^{L} G$.

Now (in the hope of gaining some insight into this mystery) we would like to formulate a geometric analogue of this relationship. The first step is to develop a geometric version of the Satake isomorphism.

5.3. Categorification of the spherical Hecke algebra. Let us look at the isomorphism of Theorem 5.1 more closely. It is easy to see that the elements $\lambda(t)$, where $\lambda \in P_{+}^{\vee}$, the set of dominant weights (with respect to a Borel subgroup of ${ }^{L} G$ ), are representatives of the double cosets of $G(F)$ with respect to $G(\mathcal{O})$. Therefore $\mathcal{H}$ has a basis $\left\{c_{\lambda}\right\}_{\lambda \in P_{+}^{\vee}}$, where $c_{\lambda}$ is the characteristic function of the double coset $G(\mathcal{O}) \lambda(t) G(\mathcal{O}) \subset G$. On the other hand, $\operatorname{Rep}^{L} G$ also has a basis labeled by $\lambda \in P_{+}^{\vee}$, which consists of the classes $\left[V_{\lambda}\right]$, where $V_{\lambda}$ is the irreducible representation with highest weight $\lambda$. However, under the Satake isomorphism these bases do not 
coincide. Instead, we have the following formula:

$$
H_{\lambda}=q^{-(\lambda, \rho)}\left(c_{\lambda}+\sum_{\mu \in P_{+}^{\vee} ; \mu<\lambda} a_{\lambda \mu} c_{\mu}\right), \quad a_{\lambda \mu} \in \mathbb{Z}_{+}[q],
$$

where $H_{\lambda}$ is the image of $\left[V_{\lambda}\right]$ in $\mathcal{H}$ under the Satake isomorphism. This formula has a remarkable geometric explanation.

Let us consider $\mathcal{H}$ as the algebra of functions on the quotient $G(F) / G(\mathcal{O})$ which are left invariant with respect to $G(\mathcal{O})$. In view of the Grothendieck fonctionsfaisceaux dictionary discussed in Section 4.2 it is natural to ask whether $G(F) / G(\mathcal{O})$ is the set of $\mathbb{F}_{q}-$ points of an algebraic variety, and if so, whether $H_{\lambda}$ is the function corresponding to a perverse sheaf on this variety. It turns out that this is indeed the case.

The quotient $G(F) / G(\mathcal{O})$ is the set of points of an ind-scheme Gr over $\mathbb{F}_{q}$ called the affine Grassmannian associated to $G$. Let $\mathcal{P}_{G(\mathcal{O})}$ be the category of $G(\mathcal{O})$ equivariant (pure) perverse sheaves on Gr. For each $\lambda \in P_{+}^{\vee}$ we have a finitedimensional $G(\mathcal{O})$-orbit $\mathrm{Gr}_{\lambda}=G(\mathcal{O}) \cdot \lambda(t)$ in Gr. Let $\mathrm{IC}_{\lambda}$ be the irreducible perverse sheaf obtained by the Goresky-MacPherson extension from the constant sheaf on $\mathrm{Gr}_{\lambda}$ to its closure $\overline{\mathrm{Gr}}_{\lambda}$. These are the irreducible objects of the category $\mathcal{P}_{G(\mathcal{O})}$.

Assigning to a perverse sheaf its "trace of Frobenius" function, we obtain an identification between the Grothendieck group of $\mathcal{P}_{G(\mathcal{O})}$ and the algebra of $G(\mathcal{O})$ invariant functions on $G(F) / G(\mathcal{O})$, i.e., the spherical Hecke algebra. In that sense, $\mathcal{P}_{G(\mathcal{O})}$ is a categorification of the Hecke algebra. A remarkable fact is that the function $H_{\lambda}$ in formula (5.2) is equal to the function associated to the perverse sheaf $\mathrm{IC}_{\lambda}$, up to a sign $(-1)^{2(\lambda, \rho)}$. Thus, under the Satake isomorphism the classes of irreducible representations of ${ }^{L} G$ go not to functions which correspond to constant sheaves on the orbits (i.e., the functions $c_{\lambda}$ ) but to the irreducible perverse sheaves. This suggests that the Satake isomorphism itself may be elevated from the level of Grothendieck groups to the level of categories. This is indeed true.

In fact, it is possible to define the structure of tensor category on $\mathcal{P}_{G(\mathcal{O})}$ with the tensor product given by a convolution functor corresponding to the convolution product (5.1) at the level of functions. Then up to a small subtlety, which has to do with the appearance of the sign $(-1)^{2(\lambda, \rho)}$ mentioned above, we have the following beautiful result. It has been conjectured by Drinfeld and proved by I. Mirković and K. Vilonen [MV] and V. Ginzburg Gi] (some important results in this direction were obtained earlier by G. Lusztig [Lu] $)$.

Theorem 5.2. The tensor category $\mathcal{P}_{G(\mathcal{O})}$ is equivalent to the tensor category of finite-dimensional representations of the group ${ }^{L} G\left(\overline{\mathbb{Q}}_{\ell}\right)$.

Moreover, the fiber functor from $\mathcal{P}_{G(\mathcal{O})}$ to the category of vector spaces (corresponding to the forgetful functor from the category of representations) is the global cohomology functor $\mathcal{F} \mapsto \oplus_{i} H^{i}(\mathrm{Gr}, \mathcal{F})$. This allows one to reconstruct the Langlands dual group ${ }^{L} G$ by means of the standard Tannakian formalism. So we get a completely new perspective on the nature of the dual group. For example, the dual group to $G L_{n}$ now appears as the group of automorphisms of the total cohomology space of the projectivization of its $n$-dimensional defining representation. This is a good illustration of why geometry is useful in the Langlands Program.

The above theorem should be viewed as a categorification of the Satake isomorphism of Theorem 5.1 We can use it to define the notion of a Hecke eigensheaf for 
an arbitrary reductive group and to formulate a geometric version of the Langlands correspondence. In the next section we do that for curves over $\mathbb{C}$, but one can apply the same technique over the finite field as well.

\section{The geometric Langlands Conjecture over $\mathbb{C}$}

In this section we will formulate the geometric Langlands conjecture for an arbitrary reductive group over $\mathbb{C}$. We will then give a brief overview of the recent work of A. Beilinson and V. Drinfeld in which a substantial part of this conjecture has been proved. It is interesting that their work uses results from representation theory of affine Kac-Moody algebras $[\mathrm{FF}],[\mathrm{Fr}$, which now play the role of the reductive groups over local non-archimedian fields.

6.1. Hecke eigensheaves. In the rest of this paper $X$ will be a smooth connected projective curve over $\mathbb{C}$ and $G$ a reductive algebraic group over $\mathbb{C}$. The results of Section 5.3 are applicable in this context. Namely, we have the affine Grassmannian over $\mathbb{C}$ and the category $\mathcal{P}_{G(\mathcal{O})}$ of $G(\mathcal{O})$-equivariant perverse sheaves (of $\mathbb{C}$-vector spaces) on Gr. This category is equivalent, as a tensor category, to the category of finite-dimensional representations of the Langlands dual group ${ }^{L} G(\mathbb{C})$. Under this equivalence, the irreducible representation of ${ }^{L} G$ with highest weight $\lambda \in P_{+}^{\vee}$ corresponds to the irreducible perverse sheaf $\mathrm{IC}_{\lambda}$.

Now we can define the analogues of the $G L_{n}$ Hecke functors introduced in Section 4.3 for general reductive groups. Let $\mathrm{Bun}_{G}$ be the moduli stack of $G$-bundles on $X$. Consider the stack $\mathcal{H}$ ecke which classifies quadruples $\left(\mathcal{M}, \mathcal{M}^{\prime}, x, \beta\right)$, where $\mathcal{M}$ and $\mathcal{M}^{\prime}$ are $G$-bundles on $X, x \in X$, and $\beta$ is an isomorphism between the restrictions of $\mathcal{M}$ and $\mathcal{M}^{\prime}$ to $X \backslash x$. We have natural morphisms

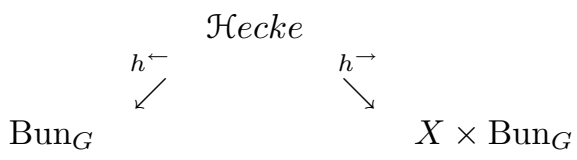

where $h^{\leftarrow}\left(\mathcal{M}, \mathcal{M}^{\prime}, x, \beta\right)=\mathcal{M}$ and $h^{\rightarrow}\left(\mathcal{M}, \mathcal{M}^{\prime}, x, \beta\right)=\left(x, \mathcal{M}^{\prime}\right)$.

Note that the fiber of $\mathcal{H}$ ecke over $\left(x, \mathcal{M}^{\prime}\right)$ is the moduli space of pairs $(\mathcal{M}, \beta)$, where $\mathcal{M}$ is a $G$-bundle on $X$, and $\beta:\left.\left.\mathcal{M}^{\prime}\right|_{X \backslash x} \stackrel{\sim}{\rightarrow} \mathcal{M}\right|_{X \backslash x}$. It is known that this is a twist of $\mathrm{Gr}_{x}=G\left(F_{x}\right) / G\left(\mathcal{O}_{x}\right)$ by the $G(\mathcal{O})_{x}$-torsor $\mathcal{M}^{\prime}\left(\mathcal{O}_{x}\right)$ of sections of $\mathcal{M}^{\prime}$ over $\operatorname{Spec} \mathcal{O}_{x}$ :

$$
\left(h^{\rightarrow}\right)^{-1}\left(x, \mathcal{M}^{\prime}\right)=\mathcal{M}^{\prime}\left(\mathcal{O}_{x}\right) \underset{G\left(\mathcal{O}_{x}\right)}{\times} \mathrm{Gr}_{x} .
$$

Therefore we have a stratification of each fiber, and hence of the entire $\mathcal{H} e c k e$, by the substacks $\mathcal{H e c k e}_{\lambda}, \lambda \in P_{+}^{\vee}$, which correspond to the $G(\mathcal{O})-$ orbits $\operatorname{Gr}_{\lambda}$ in Gr. Consider the irreducible perverse sheaf on $\mathcal{H}$ ecke, which is the Goresky-MacPherson extension of the constant sheaf on $\mathcal{H} e c k e_{\lambda}$. Its restriction to each fiber is isomorphic to $\mathrm{IC}_{\lambda}$, and by abuse of notation we will denote this entire sheaf also by $\mathrm{IC}_{\lambda}$.

Define the Hecke functor $\mathrm{H}_{\lambda}$ from the derived category of perverse sheaves on $\operatorname{Bun}_{G}$ to the derived category of perverse sheaves on $X \times$ Bun $_{G}$ by the formula

$$
\mathrm{H}_{\lambda}(\mathcal{F})=h_{!}\left(h^{\leftarrow *}(\mathcal{F}) \otimes \mathrm{IC}_{\lambda}\right) .
$$

Let $E$ be a ${ }^{L} G$-local system on $X$. Then for each irreducible representation $V_{\lambda}$ of

${ }^{L} G$ we have a local system $V_{\lambda}^{E}=E \underset{G}{\times} V_{\lambda}$. 
Definition 6.1. A perverse sheaf on $\operatorname{Bun}_{G}$ is a called a Hecke eigensheaf with eigenvalue $E$ if we are given isomorphisms

$$
\mathrm{H}_{\lambda}(\mathcal{F}) \simeq V_{\lambda}^{E} \otimes \mathcal{F},
$$

which are compatible with the tensor product structure on the category of representations of ${ }^{L} G$.

In the case when $G=G L_{n}$ this definition is equivalent to equations (4.2).

Now we can state the geometric Langlands conjecture.

Conjecture 1. Let $E$ be ${ }^{L}{ }^{L} G$-local system on $X$ which cannot be reduced to a proper parabolic subgroup of ${ }^{L} G$. Then there exists a non-zero Hecke eigensheaf Aut $_{E}$ on $\operatorname{Bun}_{G}$ with the eigenvalues $E$ which is irreducible and perverse on each connected component of $\operatorname{Bun}_{G}$.

When working over $\mathbb{C}$, we may switch from perverse sheaves to $\mathcal{D}$-modules. If $V$ is a smooth variety, we consider the sheaf of differential operators on $V$ and sheaves of modules over it, which we simply refer to as $\mathcal{D}$-modules. The simplest example of a $\mathcal{D}$-module is the sheaf of sections of a vector bundle on $V$ equipped with a flat connection (we can use the flat connection to act on sections by vector fields). The sheaf of horizontal sections of this bundle is then a locally constant sheaf, and hence a perverse sheaf. One can associate to a more general $\mathcal{D}$-module a perverse sheaf in a similar way. In fact, there is an equivalence between the category of holonomic $\mathcal{D}$-modules with regular singularities on a variety $V$ and the category of perverse sheaves on $V$, called the Riemann-Hilbert correspondence (see [B2], [GM]). Therefore we may replace in the above conjecture perverse sheaves by $\mathcal{D}$-modules. In what follows we will consider this $\mathcal{D}$-module version of the geometric Langlands conjecture.

6.2. The geometric Langlands correspondence as a Fourier-Mukai transform. Consider first the case of $G=G L_{1}$. Then $\mathrm{Bun}_{1}$ is the Picard variety Pic, and in order to prove the conjecture we should attach a Hecke eigensheaf Aut $_{E}$ on Pic to each rank one local system $E$ on $X$. Let $\sigma: \pi_{1}(X) \rightarrow \mathbb{C}^{\times}$be the homomorphism corresponding to $\sigma$. It factors through the maximal abelian quotient of $\pi_{1}(X)$, i.e., $H_{1}(X, \mathbb{Z})$. But using the cup-product on $H_{1}(X, \mathbb{Z})$ we may identify it with $H^{1}(X, \mathbb{Z})$, which is equal to $\pi_{1}\left(\mathrm{Pic}_{0}\right)$. Therefore $\sigma$ gives rise to a homomorphism $\pi_{1}\left(\mathrm{Pic}_{0}\right) \rightarrow \mathbb{C}^{\times}$and hence to a rank one local system on $\mathrm{Pic}_{0}$ or, equivalently, a flat line bundle on $\mathrm{Pic}_{0}$. This flat line bundle (considered as a $\mathcal{D}$-module) is precisely the restriction of $\mathrm{Aut}_{E}$ to $\mathrm{Pic}_{0}$. It may be extended to the other components of Pic in the same way as in Section 4.4

So in the case of $G L_{1}$ the Hecke eigensheaves are actually flat line bundles. One constructs them by using the fact that rank one local systems on a curve $X$ are the same as rank one local systems on its Jacobean $\mathrm{Jac}=\mathrm{Pic}_{0}$. Note, however, that we have used the isomorphism $H_{1}(X, \mathbb{Z}) \simeq H^{1}(X, \mathbb{Z})$. Because of that, if we take $G$ to be an arbitrary torus $T$, then rank one local systems on connected components of $\operatorname{Bun}_{T}$ will correspond to ${ }^{L} T$-local systems on $X$.

One can strengthen the statement of the geometric Langlands conjecture by using the Fourier-Mukai transform. Let $\operatorname{Loc}_{1}$ be the moduli space of rank one local systems on $X$ or, equivalently, on Jac. On the product $\operatorname{Loc}_{1} \times \mathrm{Jac}$ we have the "universal flat line bundle" Aut, whose restriction to $\{E\} \times J a c$ is the flat line bundle on Jac corresponding to $E$. It has a partial flat connection along Jac. 
This enables us to define functors between the derived category of $\mathcal{O}$-modules on $\operatorname{Loc}_{1}$ and the derived category of $\mathcal{D}$-modules on Bun 1 : pulling back to $\operatorname{Loc}_{1} \times \mathrm{Jac}$, tensoring with Aut and pushing forward to the other factor. It has been shown by G. Laumon [La4] and M. Rothstein [R] that these functors give rise to mutually inverse equivalences of derived categories, up to a sign. We note that a similar equivalence holds if one replaces Jac by an arbitrary abelian variety $A$, and it generalizes the original Fourier-Mukai correspondence in which one considers $\mathcal{O}$-modules on $A$ as opposed to $\mathcal{D}$-modules.

Note that under this equivalence the skyscraper sheaf supported at the point $\{E\} \in \mathrm{Loc}_{1}$ goes to the sheaf $\mathrm{Aut}_{E}$ on Jac. So the above equivalence may be loosely interpreted as saying that any $\mathcal{D}$-module on Jac may be expressed as a "direct integral" of the Hecke eigensheaves Aut ${ }_{E}$. In other words, the FourierMukai equivalence may be viewed as a "spectral decomposition" of the derived category of $\mathcal{D}$-modules on Jac.

Optimistically, one may hope that the Langlands correspondence for general reductive groups also gives a kind of spectral decomposition of the derived category of $\mathcal{D}$-modules on $\operatorname{Bun}_{G}$ (or, more precisely, its connected component). Namely, one may hope that there exists an equivalence between this derived category and the derived category of $\mathcal{O}$-modules on the moduli stack $\operatorname{Loc}_{L_{G}}$ of ${ }^{L} G$-local systems on $X$, so that the skyscraper sheaf on $\operatorname{Loc}_{L} G$ supported at the local system $E$ corresponds to the Hecke eigensheaf Aut $E_{E}$. If this is true, it would mean that Hecke eigensheaves provide a good "basis" in the category of $\mathcal{D}$-modules on $\operatorname{Bun}_{G}$, just as flat line bundles provide a good "basis" in the category of $\mathcal{D}$-modules on Jac.

While it is not known whether this non-abelian Fourier-Mukai transform exists, A. Beilinson and V. Drinfeld have recently constructed a special case of this transform for an arbitrary semi-simple group $G$. Roughly speaking, they construct $\mathcal{D}$-modules on $\operatorname{Bun}_{G}$ corresponding to $\mathcal{O}$-modules supported on a certain affine subvariety in $\operatorname{Loc}_{L} G$ called the space of ${ }^{L} G$-opers. Before discussing their construction, we consider its analogue in the abelian case.

6.3. A special case of Fourier-Mukai transform. The moduli space $\operatorname{Loc}_{1}$ of flat line bundles on $X$ fibers over $\mathrm{Jac}=\mathrm{Pic}_{0}$ with the fiber over $\mathcal{L} \in$ Jac being the space of all (holomorphic) connections on $\mathcal{L}$. This is an affine space over the space $H^{0}(X, \Omega)$ of holomorphic one-forms on $X$. In particular, the fiber over the trivial line bundle $\mathcal{O}$ is just the space $H^{0}(X, \Omega)$. As we have seen above, each point $\omega \in H^{0}(X, \Omega)$ gives rise to a flat line bundle on Jac. It turns out that we can describe the $\mathcal{D}$-module of sections of this flat line bundle quite explicitly.

Observe that because the tangent bundle to Jac is trivial, with the fiber isomorphic to $H^{1}(X, \Omega)$, the algebra $D$ of global differential operators on Jac is commutative and is isomorphic to $\operatorname{Sym} H^{1}(X, \mathcal{O})=$ Fun $H^{0}(X, \Omega)$ by the Serre duality. Therefore each point $\omega \in H^{0}(X, \Omega)$ gives rise to a character $\chi_{\omega}: D \rightarrow \mathbb{C}$. Define the $\mathcal{D}$-module $\mathcal{F}_{\omega}$ on Jac by the formula

$$
\mathcal{F}_{\omega}=\mathcal{D} / I_{\omega},
$$

where $\mathcal{D}$ is the sheaf of differential operators on Jac, and $I_{\omega}$ is the left ideal in $\mathcal{D}$ generated by the kernel of $\chi_{\omega}$ in $D$. Then this is a holonomic $\mathcal{D}$-module which is equal to the Hecke eigensheaf corresponding to the trivial line bundle with connection $d+\omega$ on $X$. 
We note that the $\mathcal{D}$-module $\mathcal{F}_{\omega}$ represents the system of differential equations

$$
X \cdot \Psi=\chi_{\omega}(X) \Psi, \quad X \in D,
$$

in the sense that for any homomorphism from $\mathcal{F}_{\omega}$ to another $\mathcal{D}$-module $\mathcal{K}$ the image of $1 \in \mathcal{F}_{\omega}$ in $\mathcal{K}$ is a solution of the system (6.2).

Generalizing the definition of the $\mathcal{D}$-module $\mathcal{F}_{\omega}$, we obtain a functor from the category of modules over Fun $H^{0}(X, \Omega)$ to the category of $\mathcal{D}$-modules on Jac,

$$
M \mapsto \mathcal{D} \underset{D}{\otimes} M,
$$

so that $\chi_{\omega} \mapsto \mathcal{F}_{\omega}$. This functor is the restriction of the Fourier-Mukai functor to the category of $\mathcal{O}$-modules on $\operatorname{Loc}_{1}$ supported on $H^{0}(X, \Omega) \subset \operatorname{Loc}_{1}$.

Beilinson and Drinfeld have given a similar construction for an arbitrary semisimple group $G$.

6.4. Opers. Let $G$ be a simple algebraic group, which we will assume to be connected and simply-connected in the rest of this section. Then the dual group ${ }^{L} G$ is of adjoint type. The analogue of the affine space of connections on the trivial line bundle is the space of ${ }^{L} G$-opers.

Let $G$ be a simple algebraic group of adjoint type, $B$ a Borel subgroup and $N=[B, B]$ its unipotent radical, with the corresponding Lie algebras $\mathfrak{n} \subset \mathfrak{b} \subset \mathfrak{g}$. There is an open $B$-orbit $\mathbf{O} \subset \mathfrak{n}^{\perp} / \mathfrak{b} \subset \mathfrak{g} / \mathfrak{b}$, consisting of vectors which are stabilized by the radical $N \subset B$ and such that all of their negative simple root components, with respect to the adjoint action of $H=B / N$, are non-zero. This orbit may also be described as the $B$-orbit of the sum of the projections of simple root generators $f_{i}$ of any nilpotent subalgebra $\mathfrak{n}_{-}$, which is in generic position with $\mathfrak{b}$, onto $\mathfrak{g} / \mathfrak{b}$. The torus $H=B / N$ acts simply transitively on $\mathbf{O}$, so $\mathbf{O}$ is an $H$-torsor.

Suppose we are given a principal $G$-bundle $\mathcal{F}$ on a smooth curve $X$ with a connection $\nabla$ and a reduction $\mathcal{F}_{B}$ to the Borel subgroup $B$ of $G$. Then we define the relative position of $\nabla$ and $\mathcal{F}_{B}$ (i.e., the failure of $\nabla$ to preserve $\mathcal{F}_{B}$ ) as follows. Locally, choose any flat connection $\nabla^{\prime}$ on $\mathcal{F}$ preserving $\mathcal{F}_{B}$, and take the difference $\nabla-\nabla^{\prime}$. It is easy to show that the resulting local sections of $(\mathfrak{g} / \mathfrak{b})_{\mathcal{F}_{B}} \otimes \Omega$ are independent of $\nabla^{\prime}$, and define a global $(\mathfrak{g} / \mathfrak{b})_{\mathcal{F}_{B}}$-valued one-form on $X$, denoted by $\nabla / \mathcal{F}_{B}$

Definition 6.2. $A G$-oper on $X$ is by definition a triple $\left(\mathcal{F}, \nabla, \mathcal{F}_{B}\right)$, where $\mathcal{F}$ is a principal $G$-bundle $\mathcal{F}$ on $X, \nabla$ is a connection on $\mathcal{F}$ and $\mathcal{F}_{B}$ is a $B$-reduction of $\mathcal{F}$, such that the one-form $\nabla / \mathcal{F}_{B}$ takes values in $\mathbf{O}_{\mathcal{F}_{B}} \subset(\mathfrak{g} / \mathfrak{b})_{\mathcal{F}_{B}}$.

This definition is due to Beilinson and Drinfeld [BD] (in the case when $X$ is replaced by the punctured disc Spec $\mathbb{C}((t))$ opers were introduced earlier by Drinfeld and Sokolov in their work on the generalized KdV hierarchies [DS]). Note that $\mathbf{O}$ is $\mathbb{C}^{\times}$-invariant, so that $\mathbf{O}_{\mathcal{F}_{B}}$ is well-defined in $(\mathfrak{g} / \mathfrak{b})_{\mathcal{F}_{B}}$.

For instance, in the case when $G=P G L_{n}$ this condition means that if we choose a local trivialization of $\mathcal{F}_{B}$ and a local coordinate $t$ on $X$, then the connection operator will have the form

$$
\nabla=\partial_{t}+\left(\begin{array}{ccccc}
* & * & * & \ldots & * \\
\star & * & * & \ldots & * \\
0 & \star & * & \ldots & * \\
\vdots & \ddots & \ddots & \ddots & \vdots \\
0 & 0 & \ldots & \star & *
\end{array}\right)
$$


where the $*$ 's indicate arbitrary functions in $t$ and the $\star$ 's indicate nowhere vanishing functions.

By changing the trivialization of $\mathcal{F}_{B}$ this operator may be brought in a unique way to the form

$$
\partial_{t}+\left(\begin{array}{ccccc}
0 & v_{1} & v_{2} & \cdots & v_{n-1} \\
-1 & 0 & 0 & \cdots & 0 \\
0 & -1 & 0 & \cdots & 0 \\
\vdots & \ddots & \ddots & \cdots & \vdots \\
0 & 0 & \cdots & -1 & 0
\end{array}\right)
$$

But giving such an operator is the same as giving a scalar $n$th order scalar differential operator

$$
\partial_{t}^{n}+v_{1}(t) \partial_{t}^{n-2}+\ldots+v_{n-1}(t),
$$

and a more careful calculation shows that it must act from $\Omega^{-(n-1) / 2}$ to $\left.\Omega^{(n+1) / 2}\right)$. So the space of $P G L_{n}$-opers is the space of operators of the form (6.4) (if $n$ is even, we need to choose a square root of the canonical line bundle $\Omega$, but the space of $P G L_{n}$-opers is independent of this choice). In particular, it turns out that a $P G L_{2}-$ oper is nothing but a projective connection.

Lemma 6.3. If $G$ is a simple algebraic group of adjoint type and $X$ is a smooth projective curve of genus not equal to one, then there exists a unique (up to isomorphism) G-bundle $\mathcal{F}_{0}$ which admits the structure of an oper. Moreover, the corresponding Borel reduction $\mathcal{F}_{0, B}$ is also uniquely determined, and for any connection $\nabla$ on $\mathcal{F}_{0}$ the triple $\left(\mathcal{F}_{0}, \nabla, \mathcal{F}_{0, B}\right)$ is a $G$-oper.

For example, for $G=P G L_{n}$ the bundle $\mathcal{F}_{0}$ corresponds to the rank $n$ vector bundle of $(n-1)$-jets of sections of the line bundle $\Omega^{-(n-1) / 2}$ (note that the corresponding $P G L_{n}$-bundle is independent of the choice of $\Omega^{1 / 2}$ ).

Now we switch to the Langlands dual group. The above lemma means that the space $\mathrm{Op}_{L_{G}}(X)$ of ${ }^{L} G$-opers on $X$ is an affine space which is identified with the fiber of the natural projection $\operatorname{Loc}_{L} G \rightarrow \operatorname{Bun}_{L_{G}}$ over $\mathcal{F}_{0}$.

Beilinson and Drinfeld associate to each point of $\mathrm{Op}_{L_{G}}(X)$ a Hecke eigensheaf on $\operatorname{Bun}_{G}$. Recall that in the abelian case the crucial point was that the algebra of global differential operators on Jac was isomorphic to Fun $H^{0}(X, \Omega)$. Beilinson and Drinfeld prove an analogue of this statement in the non-abelian case. However, in this case it is necessary to consider the sheaf $\mathcal{D}^{\prime}$ of differential operators acting on the square root of the canonical line bundle on $\operatorname{Bun}_{G}$ (this square root is unique up to isomorphism).

Theorem 6.4. The algebra of global sections of the sheaf $\mathcal{D}^{\prime}$ is commutative and is isomorphic to the algebra of functions on the space $\mathrm{Op}_{L} G(X)$ of ${ }^{L} G$-opers on $X$.

6.5. Hitchin's integrable system. It is instructive to look at the quasi-classical analogue of this statement. The algebra $D^{\prime}=H^{0}\left(\operatorname{Bun}_{G}, \mathcal{D}^{\prime}\right)$ carries the standard filtration by the order of the differential operator, and the associated graded algebra embeds into the algebra of functions on the cotangent bundle $T^{*} \mathrm{Bun}_{G}$ to $\mathrm{Bun}_{G}$. On the other hand, it is not difficult to show that $\mathrm{Op}_{L_{G}}(X)$ is an affine space over the space

$$
H_{G}(X)=\bigoplus_{i=1}^{\ell} H^{0}\left(X, \Omega^{\otimes\left(d_{i}+1\right)}\right),
$$


where $\ell$ is the rank of $G$, and the $d_{i}$ 's are the exponents of $G$. Therefore the algebra Fun $\mathrm{Op}_{L} G(X)$ carries a filtration such that the associated graded algebra is Fun $H_{G}$. The quasi-classical analogue of the isomorphism of Theorem 6.4 is an isomorphism Fun $T^{*} \operatorname{Bun}_{G} \simeq$ Fun $H_{G}$.

To construct such an isomorphism, we need a morphism $p: T^{*} \operatorname{Bun}_{G} \rightarrow H_{G}$. Such a morphism has been constructed by N. Hitchin Hi]. Namely, let $\mathfrak{g}$ be the Lie algebra of $G$. It is well-known that the algebra of invariant functions on $\mathfrak{g}^{*}$ is isomorphic to the graded polynomial algebra $\mathbb{C}\left[P_{1}, \ldots, P_{\ell}\right]$, where $\operatorname{deg} P_{i}=d_{i}+1$. Let us observe that the tangent space to $\operatorname{Bun}_{G}$ at $\mathcal{M} \in \operatorname{Bun}_{G}$ is isomorphic to $H^{1}\left(X, \mathfrak{g}_{\mathcal{M}}\right)$, where $\mathfrak{g}_{\mathcal{M}}=\mathcal{M} \underset{G}{\times} \mathfrak{g}$. Hence the cotangent space at $\mathcal{M}$ is isomorphic to $H^{0}\left(X, \mathfrak{g}_{\mathcal{M}}^{*} \otimes \Omega\right)$ by the Serre duality.

By definition, the Hitchin map $p$ takes $(\mathcal{M}, \eta) \in T^{*} \operatorname{Bun}_{G}$, where

$$
\eta \in H^{0}\left(X, \mathfrak{g}_{\mathcal{M}}^{*} \otimes \Omega\right)
$$

to $\left(P_{1}(\eta), \ldots, P_{\ell}(\eta)\right) \in H_{G}$. It has been proved in $[\mathrm{Hi}$, $\mathrm{Fa}$ that over an open dense subset of $H_{G}$ the morphism $p$ is smooth and its fibers are proper. Therefore we obtain an isomorphism Fun $T^{*} \operatorname{Bun}_{G} \simeq$ Fun $H_{G}$. Moreover, for any $\phi, \psi \in$ Fun $H_{G}$, we have $\left\{p^{*} \phi, p^{*} \psi\right\}=0$, where $\{\cdot, \cdot\}$ is the natural Poisson structure on $T^{*} \operatorname{Bun}_{G}$ (so that $p$ gives rise to an algebraic completely integrable system). This is a precursor of the commutativity property of the global differential operators.

6.6. Beilinson-Drinfeld construction. How can we "quantize" the map p, i.e., construct an algebra homomorphism Fun $\mathrm{Op}_{L}(X) \rightarrow D^{\prime}$ ?

In order to do this Beilinson and Drinfeld apply the following general construction. Suppose $\widetilde{M}=\operatorname{Spec} \widetilde{B}$ is an affine algebraic variety with an action of an algebraic group $H$. Let $K$ be a connected subgroup of $H$ and $M=\operatorname{Spec} B$ another affine variety such that $M=\widetilde{M} / K$, so that $B=\widetilde{B}^{K}=\widetilde{B}^{\mathfrak{k}}$, where $\mathfrak{k}=$ Lie $K$. Set $I=U \mathfrak{h} \cdot \mathfrak{k}$, where $\mathfrak{h}=$ Lie $H$, and

$$
N(I)=\{a \in U \mathfrak{h} \mid I a \subset I\} .
$$

Then $N(I) / I$ acts on $B$, and hence we obtain a homomorphism $N(I) / I \rightarrow$ $H^{0}\left(M, \mathcal{D}_{M}\right)$.

The algebra $N(I) / I$ may also be described as follows. Consider the induced $\mathfrak{h}$-module $V=\operatorname{Ind}_{\mathfrak{k}}^{\mathfrak{h}} \mathbb{C}$. Then

$$
N(I) / I=V^{\mathfrak{k}}=\left(\operatorname{End}_{\mathfrak{h}} V\right)^{\mathrm{opp}} .
$$

In our case we let $M$ be $\operatorname{Bun}_{G}$. Let $x$ be a point of $X$. In the case when the group $G$ is semi-simple, any $G$-bundle on $X$ may be trivialized already on $X \backslash x$, and so one has the following stronger version of Lemma 3.1 .

$$
\operatorname{Bun}_{G} \simeq G(\mathbb{C}[X \backslash x]) \backslash G\left(F_{x}\right) / G\left(\mathcal{O}_{x}\right) .
$$

Let

$$
\widetilde{\operatorname{Bun}}_{G}=G(\mathbb{C}[X \backslash x]) \backslash G\left(F_{x}\right)
$$

be the moduli space of pairs $(\mathcal{M}, s)$, where $\mathcal{M}$ is a $G$-bundle on $X$ and $s$ is its trivialization on the formal disc $D_{x}=\operatorname{Spec} \mathcal{O}_{x}$. We may then set $\widetilde{M}=\widetilde{\operatorname{Bun}_{G}}$, $H=G\left(F_{x}\right)$ and $K=G\left(\mathcal{O}_{x}\right)$. Though $\operatorname{Bun}_{G}$ is an algebraic stack, the above general construction is still applicable (see $[\mathrm{BD}]$ ), and so we obtain a homomorphism

$$
\left(\operatorname{End}_{\mathfrak{g}\left(F_{x}\right)} V\right)^{\mathrm{opp}} \rightarrow H^{0}\left(\operatorname{Bun}_{G}, \mathcal{D}\right) .
$$


Unfortunately, $\operatorname{End}_{\mathfrak{g}\left(F_{x}\right)} V=\mathbb{C}$, so we cannot obtain any non-trivial global differential operators on $\operatorname{Bun}_{G}$. But the Lie algebra $\mathfrak{g}\left(F_{x}\right) \simeq \mathfrak{g}((t))$ has a one-dimensional universal central extension $\widehat{\mathfrak{g}}_{\kappa}$ called the affine Kac-Moody algebra:

$$
0 \rightarrow \mathbb{C} K \rightarrow \widehat{\mathfrak{g}}_{\kappa} \rightarrow \mathfrak{g}\left(F_{x}\right) \rightarrow 0
$$

(see $[\mathrm{Kac}]$ ). As a vector space it splits into a direct sum $\mathfrak{g}\left(F_{x}\right) \oplus \mathbb{C} K$, and the commutation relations read

$$
[A \otimes f, B \otimes g]=[A, B] \otimes f g-(\kappa(A, B) \operatorname{Res} f d g) K, \quad[K, \cdot]=0,
$$

where $\kappa$ is a non-degenerate invariant inner product on $\mathfrak{g}$ (which is unique up to a scalar).

Consider the $\widehat{\mathfrak{g}}_{\kappa}$-module $V_{\kappa}=\operatorname{Ind}_{\mathfrak{g}\left(\mathcal{O}_{x}\right) \oplus \mathbb{C} K}^{\widehat{\widehat{\mathfrak{g}}}_{\kappa}} \mathbb{C}_{1}$, where $\mathbb{C}_{1}$ is the one-dimensional representation of $\mathfrak{g}\left(\mathcal{O}_{x}\right) \oplus \mathbb{C} K$ on which $\mathfrak{g}\left(\mathcal{O}_{x}\right)$ acts by 0 and $K$ acts as the identity. Then one can generalize the above construction and obtain a homomorphism from

$$
\left(\operatorname{End}_{\widehat{\mathfrak{g}}_{\kappa}} V_{\kappa}\right)^{\mathrm{opp}} \rightarrow \Gamma\left(\operatorname{Bun}_{G}, \mathcal{D}_{\kappa}\right),
$$

where $\mathcal{D}_{\kappa}$ is the sheaf of twisted differential operators corresponding to $\kappa$. In particular, if we choose the inner product $\kappa_{c}$ defined by the formula

$$
\kappa_{c}(A, B)=-\frac{1}{2} \operatorname{Tr}_{\mathfrak{g}} \text { ad } A \text { ad } B,
$$

then $\mathcal{D}_{\kappa_{c}}=\mathcal{D}^{\prime}$, the sheaf of differential operators acting on the square root of the canonical line bundle on $\operatorname{Bun}_{G}$.

The following result is due to $\mathrm{B}$. Feigin and the author $[\mathrm{FF},[\mathrm{Fr}]$.

Theorem 6.5. If $\kappa \neq \kappa_{c}$, then ${\text { End } \widehat{\mathfrak{g}}_{\kappa}}_{V_{\kappa}}=\mathbb{C}$. If $\kappa=\kappa_{c}$, then there is a canonical algebra isomorphism

$$
\operatorname{End}_{\widehat{\mathfrak{g}}_{\kappa_{c}}} V_{\kappa_{c}} \simeq{\operatorname{Fun~} \mathrm{Op}_{L} G}\left(D_{x}\right)
$$

where $D_{x}=\operatorname{Spec} \mathcal{O}_{x}$

Thus, we obtain a homomorphism $\varphi_{x}:$ Fun $\mathrm{Op}_{L_{G}}\left(D_{x}\right) \rightarrow D^{\prime}$, where $D^{\prime}$ is the algebra of global differential operators acting on the square root $\omega^{1 / 2}$ of the canonical line bundle on $\operatorname{Bun}_{G}$. Beilinson and Drinfeld prove the following theorem [BD].

Theorem 6.6. The homomorphism $\varphi_{x}$ factors through a homomorphism

$$
\varphi: \text { Fun } \mathrm{Op}_{L} G(X) \rightarrow D^{\prime}
$$

which is independent of $x$ and is an algebra isomorphism.

This proves Theorem 6.4. Now, given a ${ }^{L} G$-oper $\rho=\left(\mathcal{F}, \nabla, \mathcal{F}_{B}\right)$ on $X$, we construct a $\mathcal{D}$-module $\Delta_{\rho}$ on $\operatorname{Bun}_{G}$ :

$$
\Delta_{\rho}=\left(\mathcal{D}^{\prime} / I_{\rho}\right) \otimes \omega^{-1 / 2},
$$

where $I_{\rho}$ is the left ideal in $\mathcal{D}^{\prime}$ generated by the kernel of the character $\chi_{\rho}: D^{\prime} \rightarrow$ $\mathbb{C}$ corresponding to the point $\rho \in \operatorname{Spec} D^{\prime}$ (compare with formula (6.1)). Since $\operatorname{dim} \mathrm{Op}_{L_{G}}(X)=\operatorname{dim} \mathrm{Bun}_{G}$, the $\mathcal{D}$-module $\Delta_{\rho}$ is holonomic (it is also non-zero). Denote by $E_{\rho}$ the ${ }^{L} G$-local system $(\mathcal{F}, \nabla)$ underlying the ${ }^{L} G$-oper $\rho$. Beilinson and Drinfeld prove the following fundamental result.

Theorem 6.7. The $\mathcal{D}$-module $\Delta_{\rho}$ is a Hecke eigensheaf with eigenvalue $E_{\rho}$. 
Thus, Beilinson and Drinfeld prove the geometric Langlands Conjecture for those ${ }^{L} G$-local systems on $X$ which admit the structure of an oper.

More generally, as in Section 6.3 we obtain a functor from the category of modules over the algebra Fun $\mathrm{Op}_{L_{G}}(X)$ to the category of $\mathcal{D}$-modules on $\mathrm{Bun}_{G}$ :

$$
M \mapsto\left(\mathcal{D}^{\prime} \underset{D^{\prime}}{\otimes} M\right) \otimes \omega^{-1 / 2},
$$

so that $\chi_{\rho} \mapsto \Delta_{\rho}$. This functor may be viewed as the restriction of the wouldbe non-abelian Fourier-Mukai transform to the category of $\mathcal{O}$-modules on $\operatorname{Loc}_{L_{G}}$ supported on $\mathrm{Op}_{L} G(X) \subset \operatorname{Loc}_{L}$.

\section{REFERENCES}

[A1] J. Arthur, Automorphic representations and number theory in Seminar on Harmonic Analysis (Montreal, 1980), pp. 3-51, CMS Conf. Proc. 1, AMS, 1981. MR 84e:12012

[A2] J. Arthur, The principle of functoriality, Bull. AMS 40 (2003) 39-53.

[BBD] A. Beilinson, J. Bernstein, P. Deligne, Faisceaux pervers, Astérisque 100 (1982). MR 86g:32015

[BD] A. Beilinson and V. Drinfeld, Quantization of Hitchin's integrable system and Hecke eigensheaves, Preprint, available at www.math.uchicago.edu/ benzvi.

[B1] A. Borel, Automorphic L-functions, in Automorphic forms, representations and $L-$ functions, Proc. Symp. Pure Math. 33, Part 2, pp. 27-61, AMS, 1979. MR 81m:10056

[B2] A. Borel et al., Algebraic D-modules, Academic Press, 1987. MR 89g:32014

[C] H. Carayol, Preuve de la conjecture de Langlands locale pour $\mathrm{GL}_{n}$ : travaux de HarrisTaylor et Henniart, Séminaire Bourbaki, Exp. No. 857, Astérisque 266 (2000) 191-243. MR 2001i: 11136

[CPS] J.W. Cogdell and I.I. Piatetski-Shapiro, Converse theorems for $G L_{n}$, Publ. IHES 79 (1994) 157-214. MR 95m:22009

[De1] P. Deligne, La conjecture de Weil. I, Publ. IHES 43 (1974) 273-307. MR 49:5013

[De2] P. Deligne, La conjecture de Weil. II, Publ. IHES 52 (1980) 137-252. MR 83c:14017

[Dr1] V.G. Drinfeld, Langlands conjecture for GL(2) over function field, Proc. of Int. Congress of Math. (Helsinki, 1978), Acad. Sci. Fennica, 1980, pp. 565-574. MR 81j:12007

[Dr2] V.G. Drinfeld, Moduli varieties of F-sheaves, Funct. Anal. Appl. 21 (1987) 107-122. MR 89b:11092

[Dr3] V.G. Drinfeld, The proof of Petersson's conjecture for GL(2) over a global field of characteristic p, Funct. Anal. Appl. 22 (1988) 28-43. MR 90c:11038

[Dr4] V.G. Drinfeld, Two-dimensional $\ell$-adic representations of the fundamental group of a curve over a finite field and automorphic forms on GL(2), Amer. J. Math. 105 (1983) 85-114. MR 84i:12011

[DS] V. Drinfeld and V. Sokolov, Lie algebras and KdV type equations, J. Sov. Math. 30 (1985) $1975-2036$.

[Fa] G. Faltings, Stable G-bundles and projective connections, J. Alg. Geom. 2 (1993) 507-568. MR 94i:14015

[FF] B. Feigin and E. Frenkel, Affine Kac-Moody algebras at the critical level and Gelfand-Dikii algebras, Int. Jour. Mod. Phys. A7, Supplement 1A (1992) 197-215. MR 93j:17049

[FK] E. Freitag and R. Kiehl, Etale Cohomology and the Weil conjecture, Springer-Verlag, 1988. MR 89f:14017

[Fr] E. Frenkel, Lectures on Wakimoto modules, opers and the center at the critical level, Preprint math.QA/0210029.

[FGKV] E. Frenkel, D. Gaitsgory, D. Kazhdan, K. Vilonen, Geometric realization of Whittaker functions and the Langlands correspondence, Journal of AMS 11 (1998) 451-484. MR 99f:11148

[FGV] E. Frenkel, D. Gaitsgory, K. Vilonen, On the geometric Langlands conjecture, Journal of AMS 15 (2002) 367-417. MR 2003a:11145

[Ga] D. Gaitsgory, On a vanishing conjecture appearing in the geometric Langlands correspondence, Preprint math.AG/0204081.

[Ge] S. Gelbart, An elementary introduction to the Langlands program, Bull. Amer. Math. Soc. 10 (1984) 177-219 MR 85e:11094 
[GM] S.I. Gelfand and Yu.I. Manin, Homological algebra, Encyclopedia of Mathematical Sciences 38, Springer-Verlag, 1994. MR 95g:18007

[Gi] V. Ginzburg, Perverse sheaves on a loop group and Langlands duality, Preprint alggeom/9511007.

[Go] D. Goss, What is a Shtuka?, Notices of AMS 51 (2003) 36-37.

[Gr] A. Grothendieck, Formule de Lefschetz et rationalité des fonctions L, Séminaire Bourbaki, Exp. No. 279, Astérisque 9 (1995) 41-55.

[HK] G. Harder and D.A. Kazhdan, Automorphic forms on $\mathrm{GL}_{2}$ over function fields (after V. G. Drinfeld), in Automorphic forms, representations and $L$-functions, Proc. Symp. Pure Math. 33, Part 2, pp. 357-379, AMS, 1979. MR 83e:12008

[HT] M. Harris and R. Taylor, The geometry and cohomology of some simple Shimura varieties. With an appendix by Vladimir G. Berkovich, Annals of Mathematics Studies 151, Princeton University Press, 2001. MR 2002m:11050

[He] G. Henniart, Une preuve simple des conjectures de Langlands pour $\mathrm{GL}(n)$ sur un corps p-adique, Invent. Math. 139 (2000) 439-455. MR 2001e:11052

[Hi] N. Hitchin, Stable bundles and integrable systems, Duke Math. J. 54 (1987) 91-114. MR 88i:58068

[JL] H. Jacquet and R.P. Langlands, Automorphic Forms on GL(2), Lect. Notes in Math. 114, Springer-Verlag, 1970. MR 53:5481

[Kac] V.G. Kac, Infinite-dimensional Lie Algebras, 3rd Edition, Cambridge University Press, 1990. MR 92k:17038

[Kaz] D.A. Kazhdan, An introduction to Drinfeld's "Shtuka", in Automorphic forms, representations and $L$-functions, Proc. Symp. Pure Math. 33, Part 2, pp. 347-356, AMS, 1979. MR 82f:12018

[Kn] A.W. Knapp, Introduction to the Langlands program, in Representation theory and automorphic forms (Edinburgh, 1996), pp. 245-302, Proc. Symp. Pure Math. 61, AMS, 1997. MR 99d:11123

[Laf1] L. Lafforgue, Chtoucas de Drinfeld et conjecture de Ramanujan-Petersson, Astérisque 243 (1997). MR 99c:11072

[Laf2] L. Lafforgue, Chtoucas de Drinfeld et correspondance de Langlands, Invent. Math. 147 (2002) 1-241. MR 2002m:11039

[L1] R.P. Langlands, Problems in the theory of automorphic forms, in Lect. Notes in Math. 170, pp. 18-61, Springer-Verlag, 1970. MR 46:1758

[L2] R. Langlands, Where stands functoriality today?, in Representation theory and automorphic forms (Edinburgh, 1996), pp. 457-471, Proc. Symp. Pure Math. 61, AMS, 1997. MR 99c:11140

[La1] G. Laumon, Transformation de Fourier, constantes d'équations fonctionelles et conjecture de Weil, Publ. IHES 65 (1987) 131-210. MR 88g:14019

[La2] G. Laumon, Correspondance de Langlands géométrique pour les corps de fonctions, Duke Math. J. 54 (1987) 309-359. MR 88g:11086

[La3] G. Laumon, Faisceaux automorphes pour $G L_{n}$ : la première construction de Drinfeld, Preprint alg-geom/9511004 (1995).

[La4] G. Laumon, Transformation de Fourier généralisée, Preprint alg-geom/9603004.

[La5] G. Laumon, La correspondance de Langlands sur les corps de fonctions (d'après Laurent Lafforgue), Séminaire Bourbaki, Exp. No. 973, Preprint math.AG/0003131.

[La6] G. Laumon, Travaux de Frenkel, Gaitsgory et Vilonen sur la correspondance de DrinfeldLangland, Séminaire Bourbaki, Exp. No. 906, Preprint math.AG/0207078.

[LMB] G. Laumon, L. Moret-Bailly, Champs algébriques, Springer-Verlag, 2000. MR 2001f:14006

[LSR] G. Laumon, M. Rapoport, U. Stuhler, D-elliptic sheaves and the Langlands correspondence, Invent. Math. 113 (1993) 217-338. MR 96e:11077

[Lu] G. Lusztig, Singularities, character formulas, and a q-analogue of weight multiplicities, Astérisque 101 (1983) 208-229. MR 85m:17005

[Mi] J.S. Milne, Étale cohomology, Princeton University Press, 1980. MR 81j:14002

[MV] I. Mirković and K. Vilonen, Perverse sheaves on affine Grassmannians and Langlands duality, Math. Res. Lett. 7 (2000) 13-24. MR 2001h:14020

[Mu] M.R. Murty, A motivated introduction to the Langlands program, in Advances in number theory (Kingston, ON, 1991), pp. 37-66, Oxford Univ. Press, 1993. MR 96j:11157 
[PS1] I.I. Piatetski-Shapiro, Multiplicity one theorem, in Automorphic forms, representations and $L$-functions, Proc. Symp. Pure Math. 33, Part 2, pp. 209-212, AMS, 1979. MR 81m:22027

[PS2] I.I. Piatetski-Shapiro, Zeta-functions of $G L(n)$, Preprint of University of Maryland, 1976.

[R] M. Rothstein, Connections on the total Picard sheaf and the KP hierarchy, Acta Applicandae Mathematicae 42 (1996) 297-308. MR 97b:14037

[Se] J-P. Serre, Algebraic Groups and Class Fields, Springer-Verlag, 1988. MR 88i:14041

[Sh] J.A. Shalika, The multiplicity one theorem for $G L_{n}$, Ann. Math. 100 (1974) 171-193. MR 50:545

[Sp] T.A. Springer, Reductive groups, in Automorphic forms, representations and $L$-functions, Proc. Symp. Pure Math. 33, Part 1, pp. 3-27, AMS, 1979. MR 80h:20062

[W] A. Weil, Dirichlet Series and Automorphic Forms, Lect. Notes in Math. 189, SpringerVerlag, 1971.

Department of Mathematics, University of California, Berkeley, California 94720

E-mail address: frenkel@math.berkeley.edu 